\title{
Alignment between Protostellar Outflows and Filamentary Structure
}

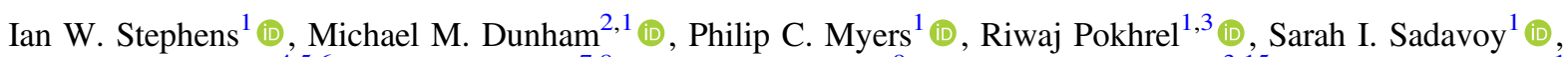 \\ Eduard I. Vorobyov ${ }^{4,5,6}$ (D) John J. Tobin ${ }^{7,8}$ (D), Jaime E. Pineda ${ }^{9}$ (D), Stella S. R. Offner ${ }^{3,15}$ (D), Katherine I. Lee ${ }^{1}$, \\ Lars E. Kristensen $^{10}$ (D), Jes K. Jørgensen ${ }^{11}$ (D), Alyssa A. Goodman ${ }^{1}$ (D), Tyler L. Bourke ${ }^{12}$, Héctor G. Arce ${ }^{13}$ (D), and \\ Adele L. Plunkett ${ }^{14}$ (iD \\ ${ }^{1}$ Harvard-Smithsonian Center for Astrophysics, 60 Garden Street, Cambridge, MA, USA; ian.stephens@cfa.harvard.edu \\ ${ }^{2}$ Department of Physics, State University of New York at Fredonia, 280 Central Avenue, Fredonia, NY 14063, USA \\ ${ }_{4}^{3}$ Department of Astronomy, University of Massachusetts, Amherst, MA 01003, USA \\ ${ }^{4}$ Institute of Fluid Mechanics and Heat Transfer, TU Wien, Vienna, A-1060, Austria \\ ${ }^{5}$ Research Institute of Physics, Southern Federal University, Stachki Ave. 194, Rostov-on-Don, 344090, Russia \\ ${ }^{6}$ University of Vienna, Department of Astrophysics, Vienna, A-1180, Austria \\ ${ }^{7}$ Homer L. Dodge Department of Physics and Astronomy, University of Oklahoma, 440 W. Brooks Street, Norman, OK 73019, USA \\ ${ }^{8}$ Leiden Observatory, Leiden University, P.O. Box 9513, 2300-RA Leiden, The Netherlands \\ ${ }^{9}$ Max-Planck-Institut für extraterrestrische Physik, Giessenbachstrasse 1, 85748 Garching, Germany \\ ${ }^{10}$ Centre for Star and Planet Formation, Niels Bohr Institute and Natural History Museum of Denmark, University of Copenhagen, \\ Øster Voldgade 5-7, DK-1350 Copenhagen K, Denmark \\ ${ }^{11}$ Niels Bohr Institute and Center for Star and Planet Formation, Copenhagen University, DK-1350 Copenhagen K., Denmark \\ ${ }^{12}$ SKA Organization, Jodrell Bank Observatory, Lower Withington, Macclesfield, Cheshire SK11 9DL, UK \\ ${ }^{13}$ Department of Astronomy, Yale University, New Haven, CT 06520, USA \\ ${ }^{14}$ European Southern Observatory, Av. Alonso de Cordova 3107, Vitacura, Santiago de Chile, Chile \\ ${ }^{15}$ Department of Astronomy, The University of Texas at Austin, Austin, TX 78712, USA \\ Received 2017 April 10; revised 2017 July 19; accepted 2017 July 22; published 2017 August 28
}

\begin{abstract}
We present new Submillimeter Array (SMA) observations of $\mathrm{CO}(2-1)$ outflows toward young, embedded protostars in the Perseus molecular cloud as part of the Mass Assembly of Stellar Systems and their Evolution with the SMA (MASSES) survey. For 57 Perseus protostars, we characterize the orientation of the outflow angles and compare them with the orientation of the local filaments as derived from Herschel observations. We find that the relative angles between outflows and filaments are inconsistent with purely parallel or purely perpendicular distributions. Instead, the observed distribution of outflow-filament angles are more consistent with either randomly aligned angles or a mix of projected parallel and perpendicular angles. A mix of parallel and perpendicular angles requires perpendicular alignment to be more common by a factor of $\sim 3$. Our results show that the observed distributions probably hold regardless of the protostar's multiplicity, age, or the host core's opacity. These observations indicate that the angular momentum axis of a protostar may be independent of the large-scale structure. We discuss the significance of independent protostellar rotation axes in the general picture of filamentbased star formation.
\end{abstract}

Key words: galaxies: star formation - ISM: clouds - ISM: jets and outflows - ISM: structure - stars: formation stars: protostars

Supporting material: figure set

\section{Introduction}

Many stars form in filamentary structures with widths of the order of 0.1 pc (e.g., Arzoumanian et al. 2011). While the exact shape of filaments is debated, e.g., cylinders versus ribbons (Auddy et al. 2016), filaments are defined by a long axis and two much shorter axes. Dense cores ( $\sim 0.1 \mathrm{pc}$ scale) either form within the filaments or form simultaneously with the filaments (Chen \& Ostriker 2015). Inhomogeneous flow or shear from colliding flows can torque cores (e.g., Fogerty et al. 2017; Clarke et al. 2017). Classically, angular momentum is expected to be hierarchically transferred from molecular clouds to cores to protostars (e.g., Bodenheimer 1995). For a star-forming filament, large-scale flows are probably either onto the short axes of the filament from its cloud (either via accretion from the cloud or accretion via a collision) or along the long filamentary axis. In a simplistic, non-turbulent scenario where one of the flows about the three filamentary axes dominates, a core will likely rotate primarily parallel or perpendicular to the parent filament. If the angular momentum direction at the protostellar scale is inherited from this core scale, the rotation axes of newly formed protostars will also be preferentially parallel or perpendicular to the filaments.

One way to empirically test the alignment between a protostar's spin and its filamentary structure is to observe a protostar's outflow direction and compare it to the filamentary structure as probed by dust emission. By using this method across five nearby star-forming regions, Anathpindika \& Whitworth (2008) found suggestive evidence that outflows (as traced by scattered light) tend to be preferentially perpendicular to filaments. On the other hand, Davis et al. (2009) found that in Orion, the orientation between outflows (as traced by $\mathrm{H}_{2}$ ) and filaments appear random. A well-focused study that analyzes the outflow-filament angles is needed to reconcile this disagreement.

The rotation axis of a protostar, or even the parent protostellar core, could also be independent of its natal filamentary structure. Some observations have shown that the angular momentum vectors of cores themselves may be randomly distributed about the sky, regardless of the cloud, 
core, or filamentary structure (Heyer 1988; Myers et al. 1991; Goodman et al. 1993; Tatematsu et al. 2016). Multiplicity could also affect rotation axes. In the Submillimeter Array (SMA, Ho et al. 2004) large project called the Mass Assembly of Stellar Systems and their Evolution with the SMA (MASSES; co-PIs: Michael Dunham and Ian Stephens), Lee et al. (2016) found that outflows of wide-binary pairs (i.e., binary pairs separated by 1000 and 10,000 au) are typically randomly aligned or perpendicular (but not parallel) to each other. Radiation-magnetohydrodynamic simulations by Offner et al. (2016) of slightly magnetically supercritical turbulent cores found the same results for wide-binary pairs. These simulations suggest that the direction of the protostellar spin axis can evolve significantly during formation, indicating that, at least for wide-binaries, the rotation axes are independent of the large-scale structure.

In this paper, we aim to observationally test whether or not a preferential alignment exists between the local filamentary elongation and the angular momentum axis as traced by outflows. To test such alignment, we use new $\mathrm{CO}$ observations from the MASSES survey to trace the molecular outflows in the Perseus molecular cloud. Along with ancillary data, we determine accurate projected outflow position angles (PAs) for 57 Class 0 and I protostars. The MASSES survey provides uniform spatial coverage of the same molecular line tracers in a single cloud, and only focuses on young sources-Class 0 and I protostars. Since these protostars are young, their parent filamentary structure has had less time to change in morphology since the birth of the stars. These outflow observations can then be compared to the filamentary structure as observed by the Herschel Gould Belt survey (e.g., André et al. 2010).

We describe the observations used in Section 2 and the outflow/filament PA extraction techniques in Section 3. We present the results in Section 4 and discuss their possible implications in Section 5. Finally, we summarize the main results in Section 6.

\section{Observations}

\subsection{Outflow and Continuum Data}

For the Perseus protostellar outflows studied in this paper, we introduce new, unpublished MASSES $\mathrm{CO}(2-1)$ data. The SMA observations were calibrated using the MIR software package $^{16}$ and imaged using the MIRIAD software package (Sault et al. 1995). More details of the data reduction process for the MASSES survey are presented in Lee et al. (2015). The new MASSES data all come from the SMA's subcompact configuration, which typically has baselines between $3 \mathrm{k} \lambda$ and $54 \mathrm{k} \lambda$, resulting in an average synthesized beam size of $\sim 3$ ". 8 . The velocity resolution of the observations is $0.26 \mathrm{~km} \mathrm{~s}^{-1}$, and the data were smoothed to $0.5 \mathrm{~km} \mathrm{~s}^{-1}$ in this study. The typical $1 \sigma \mathrm{rms}$ in a $0.5 \mathrm{~km} \mathrm{~s}^{-1}$ channel is $0.15 \mathrm{~K}$.

Along with the new MASSES CO(2-1) data, we also used new MASSES $1.3 \mathrm{~mm}$ continuum data to locate the centroid of the bipolar outflow, which is used to help measure the outflow PAs (see Section 3.1). A more detailed analysis of the continuum data will be discussed in a forthcoming paper (R. Pokhrel et al. 2017, in preparation). The SMA data will become publicly available with the MASSES data release paper (I. Stephens et al. 2017, in preparation).

\footnotetext{
16 http://www.cfa.harvard.edu/ cqi/mircook.html
}

In some cases, we use already published CO PAs (primarily from Plunkett et al. 2013 and from other MASSES data published in Lee et al. 2015, 2016) since these observations were either better quality and/or at higher resolution. These published PAs each came from observations of one of three different $J$ rotational transitions of $\mathrm{CO}$ : $\mathrm{CO}(1-0), \mathrm{CO}(2-1)$, and $\mathrm{CO}(3-2)$. The rest frequencies for these three spectral lines are $115.27120 \mathrm{GHz}$, $230.53796 \mathrm{GHz}$, and $345.79599 \mathrm{GHz}$, respectively.

The protostellar outflows analyzed in this study are presented in Table 1.

\subsection{Herschel-derived Optical Depth Maps}

Herschel is well-suited for finding filaments in Perseus given its resolution and wavelength range. The resolution at the longest Herschel wavelength $(500 \mu \mathrm{m})$ is $36^{\prime \prime}$ or $\sim 0.04 \mathrm{pc}$ at the distance of Perseus (235 pc, Hirota et al. 2008). Starforming filaments have temperatures of $\sim 10-20 \mathrm{~K}$, and thus the dust continuum will peak within the Herschel bands (70-500 $\mu \mathrm{m})$. These wavebands can be used to approximate the optical depth and the column density of Perseus filaments. Indeed, several studies have already created optical depth or column density maps of the Perseus molecular cloud using Herschel observations, including Sadavoy et al. (2014), Zari et al. (2016), and Abreu-Vicente et al. (2017). All three of the aforementioned studies assumed a modified blackbody with a specific intensity of

$$
I_{\nu}=B_{\nu}(T)\left(1-e^{-\tau_{\nu}}\right) \approx B_{\nu}(T) \tau_{\nu}
$$

where $B_{\nu}$ is the blackbody function at temperature $T$ and $\tau_{\nu}$ is the optical depth. $\tau_{\nu}$ is assumed to follow a power-law function of the form $\tau_{\nu} \propto \nu^{\beta}$, where $\beta$ is the dust emissivity index. The dust column density, $N_{\text {dust }}$, can be calculated assuming $\tau_{\nu}=N_{\text {dust }} \kappa_{\nu}$, where $\kappa_{\nu}$ is the dust opacity. Each study assumed $\tau_{\nu}$ and $T$ to be free parameters.

While these studies varied slightly, e.g., on their assumption for $\beta$, the resulting maps are very similar. We choose to use the $353 \mathrm{GHz}$ optical depth $\left(\tau_{353 \mathrm{GHz}}\right)$ map from Zari et al. (2016) since this map has been made publicly available. Zari et al. (2016) assumed a value of $\beta=2$, and they did not convert the $\tau_{353 \mathrm{GHz}}$ maps to column density. The $\tau_{353 \mathrm{GHz}}$ maps were made using only the Herschel 160, 250, 350, and $500 \mu \mathrm{m}$ maps. Each Herschel map was zero-point corrected with Planck and smoothed to the coarsest resolution $(500 \mu \mathrm{m})$, resulting in an $\tau_{353 \mathrm{GHz}}$ map at $36^{\prime \prime}$ resolution. The final $\tau_{353 \mathrm{GHz}}$ map has the pixels regridded to equatorial coordinates with pixel sizes of $18^{\prime \prime} \times 18^{\prime \prime}$. This $\tau_{353 \mathrm{GHz}}$ map also includes coarse resolution Planck $\tau_{353 \mathrm{GHz}}$ maps in the field external to the Herschel observations.

Figure 1 shows the Zari et al. (2016) $\tau_{353 \mathrm{GHz}}$ map of Perseus. For simplicity, we masked out the Planck-only regions of the map, which extend beyond the Herschel observations. The resolution of these Planck-only regions are too coarse to resolve the filaments and none of our MASSES targets are located within them.

\section{Data Analysis Techniques}

In this section, we summarize how we measure PAs for both outflows and filaments from observations. All angles are measured counterclockwise from the north celestial pole. These PAs are used to calculate the main parameter of interest, $\gamma$, which is the projected angle difference between the outflows 
Table 1

Source Information

\begin{tabular}{|c|c|c|c|c|c|c|c|}
\hline $\begin{array}{l}\text { Source } \\
\text { Name }^{a}\end{array}$ & $\begin{array}{l}\text { R.A. }^{\text {b }} \\
\text { (J2000) }\end{array}$ & $\begin{array}{l}\text { Decl. }^{\mathrm{b}} \\
\text { (J2000) }\end{array}$ & Other Names ${ }^{\mathrm{c}}$ & Region & $\begin{array}{l}\text { Multiple } \\
(\mathrm{Y} / \mathrm{N})\end{array}$ & $\begin{array}{l}T_{\text {bol }} \\
(\mathrm{K})\end{array}$ & $\begin{array}{c}\tau_{353 \mathrm{GHz}} \\
\left(\times 10^{3}\right)\end{array}$ \\
\hline Per-emb-1 & 03:43:56.806 & $+32: 00: 50.202$ & HH211-MMS & IC348 & $\mathrm{N}$ & 27 & 2.2 \\
\hline Per-emb-2 & 03:32:17.932 & $+30: 49: 47.705$ & IRAS $03292+3039$ & B1 & $\mathrm{Y}$ & 27 & 2.4 \\
\hline Per-emb-3 & 03:29:00.575 & $+31: 12: 00.204$ & $\ldots$ & NGC1333 & $\mathrm{N}$ & 32 & 1.1 \\
\hline Per-emb-5 & 03:31:20.942 & $+30: 45: 30.263$ & IRAS $03282+3035$ & $\mathrm{~B} 1$ & $\mathrm{Y}$ & 32 & 1.2 \\
\hline Per-emb-6 & 03:33:14.404 & $+31: 07: 10.715$ & $\ldots$ & B1 & $\mathrm{N}$ & 52 & 2.9 \\
\hline Per-emb-8 & 03:44:43.982 & $+32: 01: 35.210$ & $\ldots$ & IC348 & $\mathrm{Y}$ & 43 & 0.7 \\
\hline Per-emb-9 & 03:29:51.832 & $+31: 39: 05.905$ & IRAS $03267+3128$, Perseus5 & NGC1333 & $\mathrm{N}$ & 36 & 0.8 \\
\hline Per-emb-10 & 03:33:16.424 & $+31: 06: 52.063$ & $\ldots$ & $\mathrm{B} 1$ & $\mathrm{~N}$ & 30 & 3.8 \\
\hline Per-emb-11,O1 & 03:43:57.065 & $+32: 03: 04.788$ & IC348MMS & IC348 & $\mathrm{Y}$ & 30 & 1.6 \\
\hline Per-emb-11,O2 & 03:43:57.688 & $+32: 03: 09.975$ & IC348MMS & IC348 & $\mathrm{Y}$ & 30 & 1.9 \\
\hline Per-emb-12 & 03:29:10.537 & $+31: 13: 30.925$ & NGC1333 IRAS4A & NGC1333 & $\mathrm{Y}$ & 29 & 4.6 \\
\hline Per-emb-13,O1 & 03:29:12.016 & $+31: 13: 08.031$ & NGC1333 IRAS4B & NGC1333 & $\mathrm{Y}$ & 28 & 7.1 \\
\hline Per-emb-13,O2 & $03: 29: 12.842$ & $+31: 13: 06.893$ & NGC1333 IRAS4B' & NGC1333 & $\mathrm{Y}$ & 28 & 7.9 \\
\hline Per-emb-15 & 03:29:04.055 & $+31: 14: 46.237$ & RNO15-FIR & NGC1333 & $\mathrm{N}$ & 36 & 3.1 \\
\hline Per-emb-16 & 03:43:50.978 & $+32: 03: 24.101$ & $\ldots$ & IC348 & $\mathrm{Y}$ & 39 & 1.6 \\
\hline Per-emb-17 & 03:27:39.104 & $+30: 13: 03.078$ & $\ldots$ & L1455 & $\mathrm{Y}$ & 59 & 0.5 \\
\hline Per-emb-18 & $03: 29: 11.258$ & $+31: 18: 31.073$ & NGC1333 IRAS7 & NGC1333 & $\mathrm{Y}$ & 59 & 1.3 \\
\hline Per-emb-19 & 03:29:23.498 & $+31: 33: 29.173$ & $\ldots$ & NGC1333 & $\mathrm{N}$ & 60 & 1.0 \\
\hline Per-emb-20 & 03:27:43.276 & $+30: 12: 28.781$ & L1455-IRS4 & L1455 & $\mathrm{N}$ & 65 & 1.6 \\
\hline Per-emb-21 & 03:29:10.668 & $+31: 18: 20.191$ & $\ldots$ & NGC1333 & $\mathrm{Y}$ & 45 & 1.6 \\
\hline Per-emb-22 & $03: 25: 22.410$ & $+30: 45: 13.254$ & L1448-IRS2 & L1448 & $\mathrm{Y}$ & 43 & 1.1 \\
\hline Per-emb-23 & 03:29:17.211 & $+31: 27: 46.302$ & ASR 30 & NGC1333 & $\mathrm{N}$ & 42 & 1.0 \\
\hline Per-emb-24 & 03:28:45.297 & $+31: 05: 41.693$ & $\ldots$ & NGC1333 & $\mathrm{N}$ & 67 & 0.9 \\
\hline Per-emb-25 & $03: 26: 37.511$ & $+30: 15: 27.813$ & $\ldots$ & L1455 & $\mathrm{N}$ & 61 & 0.4 \\
\hline Per-emb-26 & $03: 25: 38.875$ & $+30: 44: 05.283$ & L1448C, L1448-mm & L1448 & $\mathrm{Y}$ & 47 & 1.8 \\
\hline Per-emb-27,O1 & 03:28:55.569 & $+31: 14: 37.022$ & NGC1333 IRAS2A & NGC1333 & $\mathrm{Y}$ & 69 & 1.7 \\
\hline Per-emb-27,O2 & $03: 28: 55.563$ & $+31: 14: 36.408$ & NGC1333 IRAS2A & NGC1333 & $\mathrm{Y}$ & 69 & 1.7 \\
\hline Per-emb-28 & 03:43:51.008 & $+32: 03: 08.042$ & $\ldots$ & IC348 & $\mathrm{Y}$ & 45 & 1.8 \\
\hline Per-emb-29 & 03:33:17.877 & $+31: 09: 31.817$ & B1-c & $\mathrm{B} 1$ & $\mathrm{~N}$ & 48 & 2.7 \\
\hline Per-emb-33,O1 & $03: 25: 36.380$ & $+30: 45: 14.723$ & L1448IRS3B, L1448N & L1448 & $\mathrm{Y}$ & 57 & 4.7 \\
\hline Per-emb-33,O2 & 03:25:36.499 & $+30: 45: 21.880$ & L1448IRS3B, L1448N & L1448 & $\mathrm{Y}$ & 57 & 4.8 \\
\hline Per-emb-33,O3 & 03:25:35.669 & $+30: 45: 34.110$ & L1448IRS3B, L1448N & L1448 & $\mathrm{Y}$ & 57 & 4.3 \\
\hline Per-emb-35,O1 & 03:28:37.091 & $+31: 13: 30.788$ & NGC1333 IRAS1 & NGC1333 & $\mathrm{Y}$ & 103 & 0.6 \\
\hline Per-emb-35,O2 & 03:28:37.219 & $+31: 13: 31.751$ & NGC1333 IRAS1 & NGC1333 & $\mathrm{Y}$ & 103 & 0.6 \\
\hline Per-emb-36 & 03:28:57.374 & $+31: 14: 15.765$ & NGC1333 IRAS2B & NGC1333 & $\mathrm{Y}$ & 106 & 1.6 \\
\hline Per-emb-37 & 03:29:18.965 & $+31: 23: 14.304$ & $\ldots$ & NGC1333 & $\mathrm{Y}$ & 22 & 0.8 \\
\hline Per-emb-40 & 03:33:16.669 & $+31: 07: 54.902$ & B1-a & $\mathrm{B} 1$ & $\mathrm{Y}$ & 132 & 2.0 \\
\hline Per-emb-41 & 03:33:20.341 & $+31: 07: 21.355$ & B1-b & $\mathrm{B} 1$ & $\mathrm{Y}$ & 157 & 4.1 \\
\hline Per-emb-42 & $03: 25: 39.135$ & $+30: 43: 57.909$ & L1448C-S & L1448 & $\mathrm{Y}$ & 163 & 1.9 \\
\hline Per-emb-44 & 03:29:03.766 & $+31: 16: 03.810$ & SVS 13A & NGC1333 & $\mathrm{Y}$ & 188 & 3.0 \\
\hline Per-emb-46 & 03:28:00.415 & $+30: 08: 01.013$ & $\ldots$ & L1455 & $\mathrm{N}$ & 221 & 0.8 \\
\hline Per-emb-49 & 03:29:12.953 & $+31: 18: 14.289$ & $\ldots$ & NGC1333 & $\mathrm{Y}$ & 239 & 2.3 \\
\hline Per-emb-50 & 03:29:07.768 & $+31: 21: 57.128$ & $\ldots$ & NGC1333 & $\mathrm{N}$ & 128 & 0.7 \\
\hline Per-emb-53 & 03:47:41.591 & $+32: 51: 43.672$ & B5-IRS1 & B5 & $\mathrm{N}$ & 287 & 0.8 \\
\hline Per-emb-55 & 03:44:43.298 & $+32: 01: 31.223$ & IRAS $03415+3152$ & IC348 & $\mathrm{Y}$ & 309 & 0.5 \\
\hline Per-emb-56 & 03:47:05.450 & $+32: 43: 08.240$ & IRAS $03439+3233$ & B5 & $\mathrm{N}$ & 312 & 0.4 \\
\hline Per-emb-57 & 03:29:03.331 & $+31: 23: 14.573$ & $\ldots$ & NGC1333 & $\mathrm{N}$ & 313 & 0.4 \\
\hline Per-emb-58 & 03:28:58.422 & $+31: 22: 17.481$ & $\ldots$ & NGC1333 & $\mathrm{N}$ & 322 & 1.2 \\
\hline Per-emb-61 & 03:44:21.357 & $+31: 59: 32.514$ & $\ldots$ & IC348 & $\mathrm{N}$ & 371 & 0.7 \\
\hline Per-emb-62 & 03:44:12.977 & $+32: 01: 35.419$ & $\ldots$ & IC348 & $\mathrm{N}$ & 378 & 0.4 \\
\hline SVS 13B & 03:29:03.078 & $+31: 15: 51.740$ & $\ldots$ & NGC1333 & $\mathrm{Y}$ & 20 & 2.7 \\
\hline SVS 13C & 03:29:01.970 & $+31: 15: 38.053$ & $\ldots$ & NGC1333 & $\mathrm{Y}$ & 21 & 2.5 \\
\hline B1-bN & 03:33:21.209 & $+31: 07: 43.665$ & $\ldots$ & $\mathrm{B} 1$ & $\mathrm{Y}$ & 14.7 & 4.9 \\
\hline B1-bS & $03: 33: 21.355$ & $+31: 07: 26.372$ & $\ldots$ & B1 & $\mathrm{Y}$ & 17.7 & 5.8 \\
\hline L1448IRS2E & $03: 25: 25.660$ & $+30: 44: 56.695$ & $\ldots$ & L1448 & $\mathrm{N}$ & 15 & 2.6 \\
\hline L1451-MMS & $03: 25: 10.245$ & $+30: 23: 55.059$ & $\ldots$ & L1451 & $\mathrm{N}$ & 15 & 0.9 \\
\hline Per-bolo-58 & 03:29:25.464 & $+31: 28: 14.880$ & $\ldots$ & NGC1333 & $\mathrm{N}^{\mathrm{d}}$ & 15 & 0.9 \\
\hline
\end{tabular}

Notes.

a Names including O1, O2, and $\mathrm{O} 3$ are sources with multiple outflows.

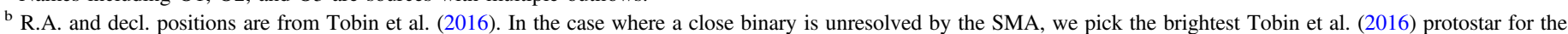
source of the emission.

${ }^{\mathrm{c}}$ Alternate names are taken from Tobin et al. (2016).

${ }^{\mathrm{d}}$ This source was not detected in Tobin et al. (2016). 


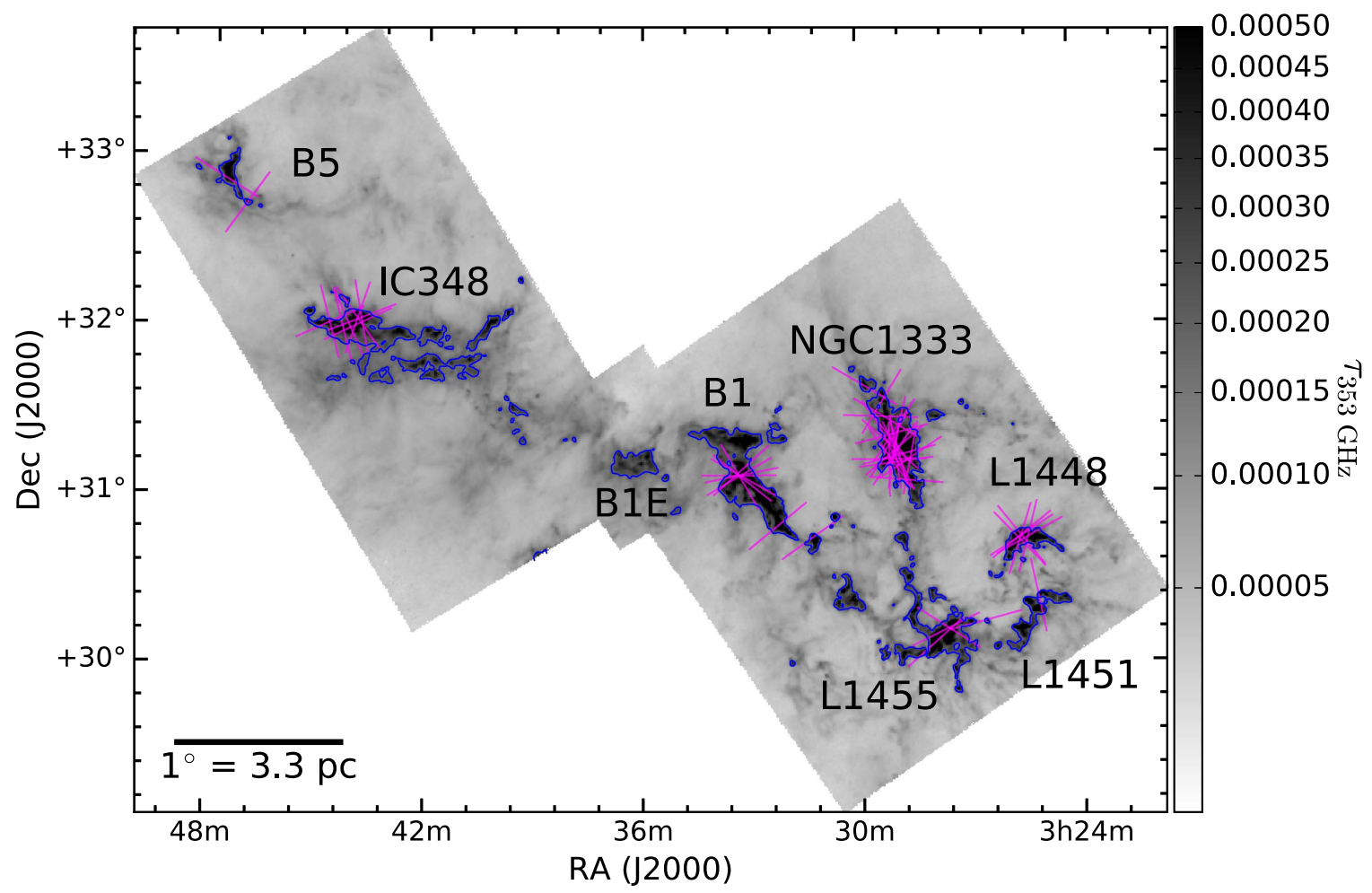

Figure 1. $\tau_{353 \mathrm{GHz}}$ map of the Perseus molecular cloud (Zari et al. 2016), with magenta lines showing the directions of the outflows measured in this study. The size of the lines only represents the direction of the outflow and not the angular extent. Thin blue contours are shown for $\tau_{353 \mathrm{GHz}}=0.0002$. These contours roughly show the boundaries of each labeled clump and correspond to a column density of $N\left(\mathrm{H}_{2}\right) \approx 5 \times 10^{21} \mathrm{~cm}^{-2}$ (Sadavoy et al. 2014).

and filaments. Specifically, $\gamma$ is given by

$$
\gamma=\operatorname{MIN}\left\{\left|\mathrm{PA}_{\text {Out }}-\mathrm{PA}_{\text {Fil }}\right|, 180^{\circ}-\left|\mathrm{PA}_{\text {Out }}-\mathrm{PA}_{\text {Fil }}\right|\right\},
$$

where $\mathrm{PA}_{\text {Out }}$ and $\mathrm{PA}_{\mathrm{Fil}}$ are the PAs of the outflow and filament, respectively. MIN indicates that we are interested in the minimum of the two values in the brackets. Table 2 lists the measured PAs for all outflows and filaments in this study.

\subsection{Outflow PAs}

We present the outflow PAs in Table 2. We independently measure the outflow PAs for both the blue- and redshifted outflows (henceforth, called the blue and red lobes). The range of the PA measurements are from $-180^{\circ}$ to $+180^{\circ}$; both positive and negative values allow one to assign the appropriate quadrant for the outflow. We also provide the combined PA, $\mathrm{PA}_{\text {Out }}$, which is simply the average of the two outflows after adding $180^{\circ}$ to the lobe with the negative PA. Some entries only provide measurements for one lobe because the other lobe was undetected.

In many cases (about half of the sources), we used outflow PAs from other $\mathrm{CO}$ line studies in place of MASSES observations since these studies had data that are better quality and/or at higher resolution. We indicate which study provided the outflow direction for each protostar in the "Ref/Info" column of Table 2. For the majority of the measured outflow PAs in this study, we made measurements using a methodology very similar to that used in Hull et al. (2013). We connect the peak intensity of the SMA $1.3 \mathrm{~mm}$ continuum observations with the peak of the integrated intensity maps for both the blue and red outflow lobes. Based on visual inspection, if the $\mathrm{CO}$ line emission obviously traces the outflow cavity walls rather than the outflow centroid, we connect the continuum peak to a local CO maximum near the continuum rather than the absolute maximum. In cases where there are no clear local outflow maxima for one lobe, we use the PA measured by the other lobe. If no local maxima exists for both lobes and the $\mathrm{CO}$ only traces cavity walls, we manually measure the PA by eye. We indicate in the "Ref/Info" column of Table 2 which outflow measuring method we used. For the angles measured in this paper, a crude approximation of the uncertainty can be found by subtracting the blue outflow PA from the red outflow PA. With such an approximation, the uncertainty in the outflow PA is typically less than $10^{\circ}$.

Frequently, the observed field about a MASSES target overlaps with other protostellar sources, which can cause significant confusion in assigning which emission comes from which protostar. To disentangle which emission belongs to which source, we used SAOImage DS9 to overlay all CO emission detected with MASSES on top of Spitzer IRAC emission (not shown). In particular, both the 3.6 and $4.5 \mu \mathrm{m}$ Spitzer bands trace the outflow cavities in scattered light and/or knots of $\mathrm{H}_{2}$ emission that are most prominent in the $4.5 \mu \mathrm{m}$ channel. We also use the catalog of Perseus protostars from Young et al. (2015) to locate other nearby $\mathrm{T}$ Tauri stars that may be contributing to the $\mathrm{CO}$ emission observed by the SMA. Together, we are able to disentangle which outflow emanates from which source. In this paper, we only present the outflow PAs that we believe we were confidently able to determine. Protostars surveyed by MASSES that are not presented in this paper were either not yet imaged or had confusing $\mathrm{CO}$ emission that did not allow for a reliable measurement of $\mathrm{PA}_{\mathrm{Out}}$. In total, we have $\mathrm{PA}_{\text {Out }}$ measurements for 57 protostellar outflows. In Figure 1, we overlay each $\mathrm{PA}_{\text {Out }}$ measurement on the Herschel-derived $\tau_{353 \mathrm{GHz}}$ map. The SMA 
Table 2

Measured Position Angles and Outflow-filament Angles

\begin{tabular}{|c|c|c|c|c|c|c|c|c|c|c|c|c|c|c|}
\hline $\begin{array}{l}\text { Source } \\
\text { Name }^{\text {a }}\end{array}$ & $\begin{array}{c}\text { Blue PA } \\
\left({ }^{\circ}\right)\end{array}$ & $\begin{array}{c}\text { Red PA } \\
\left(^{\circ}\right)\end{array}$ & $\mathrm{PA}_{\text {Out }}$ & $\begin{array}{c}\mathrm{Ref} / \mathrm{Info}^{\mathrm{b}} \\
\left(^{\circ}\right)\end{array}$ & $\begin{array}{c}\mathrm{PA}_{\mathrm{Fil}, \mathrm{F}} \\
\left(^{\circ}\right)\end{array}$ & $\begin{array}{l}\gamma_{\mathrm{F}} \\
\left(^{\circ}\right)\end{array}$ & $\begin{array}{l}\gamma_{1^{\prime}} \\
\left({ }^{\circ}\right)\end{array}$ & $\gamma_{2^{\prime}}$ & $\gamma_{3^{\prime}}$ & $\gamma_{4^{\prime}}$ & $\gamma_{5^{\prime}}$ & $\gamma_{6^{\prime}}$ & $\gamma_{\mathrm{se}, \mathrm{S}}$ & $\gamma_{\mathrm{se}, \mathrm{L}}$ \\
\hline Per-emb-1 & 114 & -61 & 116 & (1) & 40 & 76 & 82 & 35 & 25 & 24 & 19 & 21 & 79 & 39 \\
\hline Per-emb-2 & 129 & -50 & 129 & (1) & 132 & 3 & 4 & 77 & 83 & 85 & 87 & 86 & 75 & 85 \\
\hline Per-emb-3 & -82 & 95 & 97 & (2) & 10 & 87 & 87 & 82 & 75 & 73 & 64 & 38 & 47 & 77 \\
\hline Per-emb-5 & 126 & -56 & 125 & (1) & 39 & 86 & 75 & 80 & 73 & 80 & 84 & 80 & 73 & 81 \\
\hline Per-emb-6 & 50 & -109 & 60 & (1) & 48 & 12 & 14 & 11 & 11 & 11 & 9 & 11 & 15 & 16 \\
\hline Per-emb-8 & 15 & -165 & 15 & (3) & 65 & 50 & 47 & 47 & 48 & 45 & 50 & 50 & 88 & 62 \\
\hline Per-emb-9 & 63 & -125 & 59 & (1) & 54 & 5 & 2 & 1 & 1 & 3 & 77 & 14 & 27 & 39 \\
\hline Per-emb-10 & -134 & 57 & 52 & (1) & 48 & 4 & 5 & 2 & 2 & 2 & 1 & 3 & 24 & 8 \\
\hline Per-emb-11,O1 & -17 & 161 & 162 & (1) & 134 & 27 & 27 & 38 & 40 & 38 & 37 & 61 & 78 & 85 \\
\hline Per-emb-11,O2 & 36 & $\ldots$ & 36 & $(3),(7)$ & 134 & 82 & 81 & 88 & 86 & 88 & 89 & 7 & 48 & 41 \\
\hline Per-emb-12 & -145 & 35 & 35 & (2) & 128 & 87 & 87 & 85 & 85 & 0 & 9 & 80 & 82 & 15 \\
\hline Per-emb-13,O1 & 180 & 0 & 180 & (2) & 130 & 50 & 50 & 48 & 46 & 35 & 26 & 45 & 47 & 21 \\
\hline Per-emb-13,O2 & -90 & 90 & 90 & (3) & 130 & 40 & 40 & 41 & 44 & 49 & 64 & 45 & 43 & 70 \\
\hline Per-emb-15 & 145 & -35 & 145 & (2) & 42 & 77 & 77 & 82 & 87 & 4 & 61 & 14 & 12 & 56 \\
\hline Per-emb-16 & 14 & -173 & 11 & (1) & 77 & 67 & 82 & 85 & 84 & 79 & 75 & 78 & 73 & 66 \\
\hline Per-emb-17 & -127 & 60 & 57 & (1) & 146 & 89 & 90 & 81 & 82 & 86 & 83 & 83 & 65 & 90 \\
\hline Per-emb-18 & -30 & 150 & 150 & (3) & 20 & 50 & 73 & 3 & 7 & 75 & 74 & 66 & 63 & 51 \\
\hline Per-emb-19 & -32 & 148 & 148 & $(1),(8)$ & 27 & 59 & 57 & 77 & 17 & 17 & 24 & 42 & 55 & 53 \\
\hline Per-emb-20 & -61 & 112 & 115 & (1) & 58 & 58 & 18 & 15 & 26 & 27 & 29 & 28 & 6 & 32 \\
\hline Per-emb-21 & 48 & -132 & 48 & (3) & 20 & 28 & 15 & 35 & 25 & 3 & 4 & 22 & 15 & 28 \\
\hline Per-emb-22 & -62 & 118 & 118 & $(1),(8)$ & 61 & 57 & 43 & 37 & 37 & 37 & 37 & 36 & 26 & 29 \\
\hline Per-emb-23 & -125 & 61 & 58 & (1) & 138 & 79 & 17 & 17 & 45 & 43 & 46 & 85 & 56 & 38 \\
\hline Per-emb-24 & -103 & 93 & 85 & (1) & 54 & 31 & 29 & 27 & 26 & 23 & 56 & 59 & 33 & 64 \\
\hline Per-emb-25 & -78 & 107 & 104 & (1) & 61 & 43 & 31 & 45 & 64 & 47 & 48 & 10 & 84 & 43 \\
\hline Per-emb-26 & -21 & 165 & 162 & (1) & 130 & 32 & 39 & 36 & 35 & 39 & 33 & 39 & 70 & 73 \\
\hline Per-emb-27,O1 & -156 & 4 & 14 & (2) & 125 & 69 & 80 & 84 & 79 & 46 & 22 & 27 & 57 & 7 \\
\hline Per-emb-27,O2 & -77 & 105 & 104 & (2) & 125 & 21 & 10 & 6 & 11 & 44 & 68 & 63 & 33 & 84 \\
\hline Per-emb-28 & 112 & -68 & 112 & (3) & 77 & 35 & 3 & 6 & 5 & 0 & 4 & 21 & 28 & 35 \\
\hline Per-emb-29 & 133 & -50 & 132 & (1) & 7 & 55 & 58 & 57 & 62 & 48 & 80 & 7 & 20 & 88 \\
\hline Per-emb-33,O1 & -58 & 122 & 122 & $(3),(4)$ & 127 & 5 & 12 & 9 & 12 & 13 & 9 & 10 & 30 & 33 \\
\hline Per-emb-33,O2 & 38 & -142 & 38 & $(3),(4)$ & 127 & 89 & 72 & 75 & 72 & 71 & 69 & 74 & 54 & 51 \\
\hline Per-emb-33,O3 & -52 & 128 & 128 & $(3),(4)$ & 130 & 2 & 19 & 19 & 23 & 24 & 21 & 19 & 36 & 39 \\
\hline Per-emb-35,O1 & -57 & 123 & 123 & $(1),(8)$ & 32 & 89 & 68 & 73 & 86 & 86 & 89 & 85 & 67 & 78 \\
\hline Per-emb-35,O2 & 169 & -11 & 169 & $(1),(8)$ & 32 & 43 & 22 & 27 & 48 & 49 & 46 & 39 & 67 & 32 \\
\hline Per-emb-36 & -156 & $\ldots$ & 24 & $(2),(7)$ & 134 & 70 & 85 & 85 & 89 & 7 & 9 & 76 & 67 & 4 \\
\hline Per-emb-37 & -139 & 34 & 38 & (1) & 30 & 7 & 7 & 22 & 12 & 4 & 8 & 8 & 20 & 17 \\
\hline Per-emb-40 & 101 & -79 & 101 & $(1),(9)$ & 44 & 57 & 33 & 54 & 54 & 51 & 51 & 34 & 26 & 57 \\
\hline Per-emb-41 & -150 & 30 & 30 & (3) & 125 & 85 & 85 & 84 & 83 & 84 & 80 & 82 & 45 & 14 \\
\hline Per-emb-42 & 43 & -137 & 43 & (3) & 130 & 87 & 80 & 83 & 84 & 80 & 85 & 80 & 49 & 46 \\
\hline Per-emb-44 & 120 & -40 & 130 & (2) & 13 & 63 & 72 & 70 & 65 & 21 & 76 & 3 & 3 & 71 \\
\hline Per-emb-46 & -49 & 131 & 131 & (10) & 10 & 59 & 62 & 64 & 21 & 24 & 20 & 15 & 20 & 16 \\
\hline Per-emb-49 & -153 & 27 & 27 & (3) & 20 & 7 & 16 & 15 & 4 & 18 & 17 & 9 & 6 & 7 \\
\hline Per-emb-50 & -83 & 112 & 104 & (1) & 120 & 16 & 16 & 19 & 16 & 62 & 66 & 67 & 48 & 84 \\
\hline Per-emb-53 & 52 & -114 & 59 & (1) & 26 & 33 & 30 & 13 & 43 & 34 & 46 & 42 & 74 & 18 \\
\hline Per-emb-55 & 115 & -65 & 115 & (3) & 65 & 50 & 53 & 53 & 52 & 55 & 56 & 50 & 8 & 38 \\
\hline Per-emb-56 & 145 & -35 & 145 & (10) & 54 & 89 & 81 & 78 & 15 & 82 & 86 & 81 & 66 & 76 \\
\hline Per-emb-57 & 145 & 147 & 146 & $(1),(11)$ & 135 & 11 & 7 & 11 & 34 & 75 & 73 & 68 & 6 & 54 \\
\hline Per-emb-58 & -13 & $\ldots$ & 167 & $(1),(7)$ & 135 & 32 & 29 & 32 & 54 & 58 & 55 & 54 & 14 & 34 \\
\hline Per-emb-61 & 15 & -165 & 15 & $(1),(9)$ & 134 & 61 & 61 & 66 & 75 & 83 & 86 & 84 & 41 & 62 \\
\hline Per-emb-62 & -155 & 24 & 24 & (1) & 132 & 72 & 76 & 63 & 79 & 75 & 78 & 84 & 76 & 52 \\
\hline SVS 13B & $\ldots$ & -20 & 170 & $(3),(7)$ & 14 & 24 & 32 & 30 & 46 & 22 & 36 & 41 & 37 & 31 \\
\hline SVS $13 C$ & -172 & 8 & 8 & (2) & 14 & 6 & 14 & 30 & 28 & 39 & 18 & 61 & 55 & 13 \\
\hline B1-bN & 90 & $\ldots$ & 90 & (3), (7) & 128 & 38 & 37 & 38 & 39 & 38 & 43 & 38 & 45 & 76 \\
\hline B1-bS & 112 & -68 & 120 & (3) & 125 & 5 & 5 & 6 & 7 & 6 & 10 & 7 & 15 & 46 \\
\hline L1448IRS2E & $\ldots$ & 165 & 165 & $(5),(7)$ & 62 & 77 & 87 & 87 & 86 & 83 & 84 & 81 & 73 & 76 \\
\hline L1451-MMS & 11 & -169 & 11 & (6) & 123 & 68 & 71 & 37 & 71 & 60 & 61 & 34 & 54 & 53 \\
\hline Per-bolo-58 & 87 & -93 & 87 & (1) & 56 & 32 & 40 & 41 & 65 & 67 & 72 & 52 & 41 & 67 \\
\hline
\end{tabular}

Notes.

a Names including $\mathrm{O} 1, \mathrm{O} 2$, and $\mathrm{O} 3$ are sources with multiple outflows.

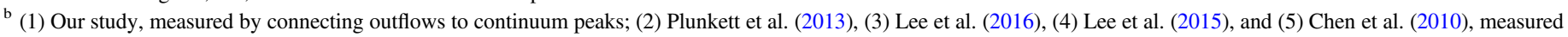

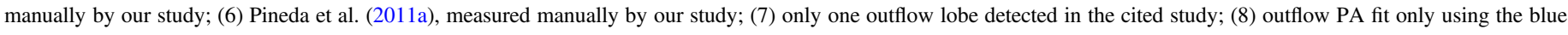

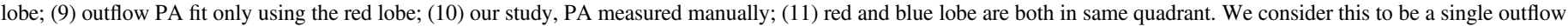
that may be in the plane of the sky. 

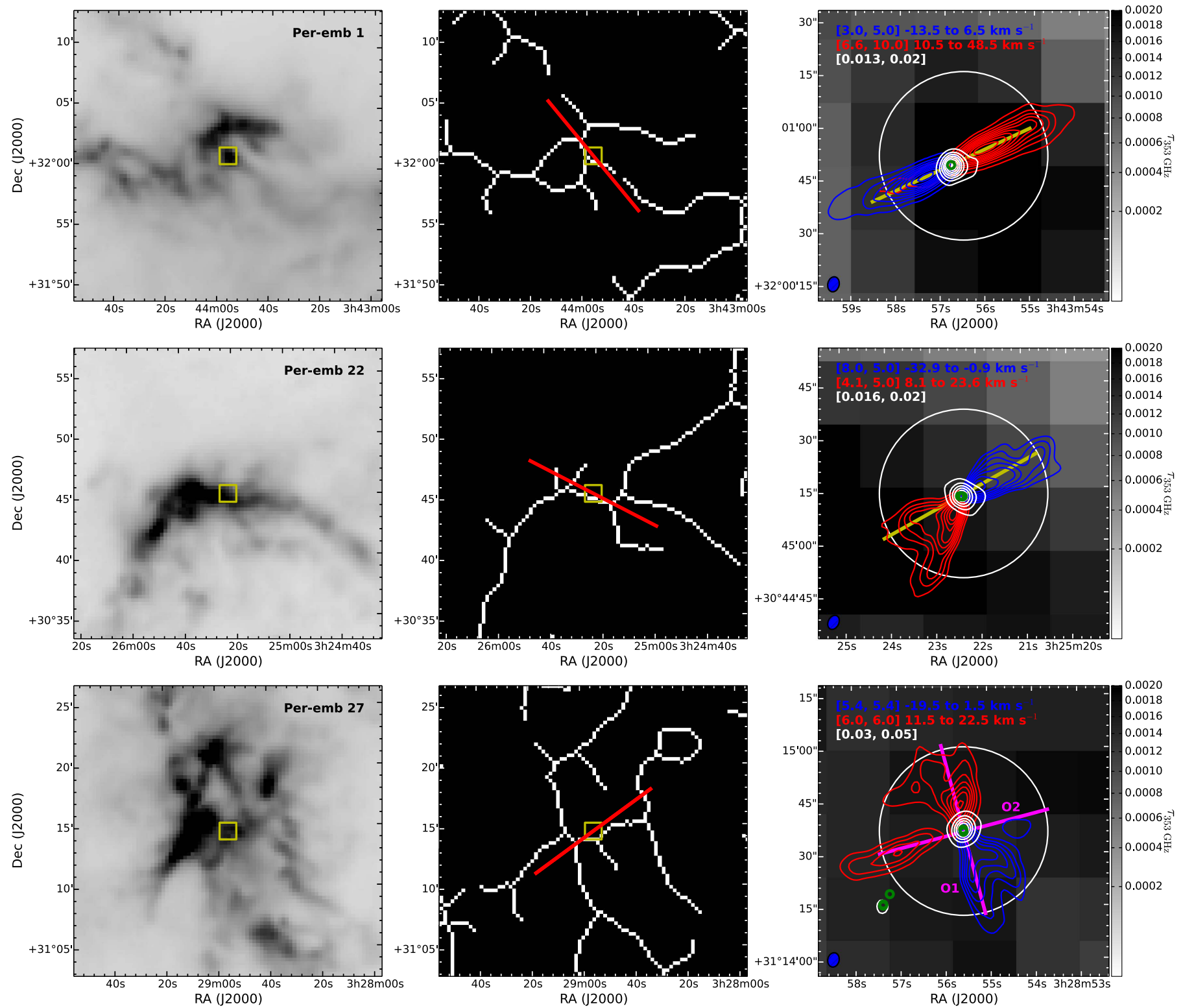

Figure 2. Figures demonstrating the FILFINDER algorithm for Per-emb 1 (top 3 panels), Per-emb 22 (middle 3 panels), and Per-emb 27 (bottom 3 panels); other Perseus protostars can be found in the figure set. The left and middle panels show the $\tau_{353 \mathrm{GHz}}$ maps (Zari et al. 2016) and the fitted filament skeletons from FILFINDER (Koch \& Rosolowsky 2015), respectively. The red line in the middle panel shows the fitted PA $\mathrm{Fil}_{\text {, F }}$ for the protostar. The yellow squares in these two panels show the area we zoom-in on for the right panels. The right panels show the $\tau_{353 \mathrm{GHz}}$ overlaid with SMA red and blue $\mathrm{CO}(2-1)$ integrated intensity contours of the red and blue lobes, respectively. The white contours show the SMA $1.3 \mathrm{~mm}$ continuum. The color-coded bracketed numbers in the top left give the first contour level followed by the contour level increment for each subsequent contour. The $\mathrm{CO}(2-1)$ contour levels and increments are in units of Jy beam ${ }^{-1} \mathrm{~km} \mathrm{~s}^{-1}$, while the continuum contour levels and increments are in units of $\mathrm{Jy} \mathrm{beam}^{-1}$. The red and blue velocity interval for $\mathrm{CO}(2-1)$ intensity integration are shown next to their corresponding contour levels. The small green circles show the location of the protostellar sources as determined at high resolution by the VLA (Tobin et al. 2016). The measured $\mathrm{PA}_{\mathrm{Out}}$ is shown as a line under the contours, and the line is yellow if $\mathrm{PA}_{\text {Out }}$ comes from this study, and magenta if $\mathrm{PA}_{\text {Out }}$ comes from other studies (as indicated in Table 2). The white circle shows the $48^{\prime \prime}$ diameter (FWHM) primary beam of the SMA.

(The complete figure set (45 images) is available.)

$\mathrm{CO}(2-1)$ integrated intensity maps for two protostars are shown in the right panels of Figure 2; other sources can be found in the Figure set. The average spectra within the vicinity of the protostar (i.e., within a radius of $8^{\prime \prime}$ ) is shown in Figure 3.

\subsection{Filament Direction}

We present the filament PAs in Table 2. We determine the filament directions based on Herschel-derived $\tau_{353 \mathrm{GHz}}$ maps (see Section 2.2). Since extracting directions can sometimes depend on the method used, we use two different techniques. One technique is based on FILFINDER and the other is based on SExtractor. For both techniques, we also investigate how the filament directions depend on both small- and large-scale optical depth characteristics.

\subsubsection{Using FILFINDER for Filament PAs}

The first method extracts the filamentary structure using the FILFINDER algorithm (Koch \& Rosolowsky 2015) as 

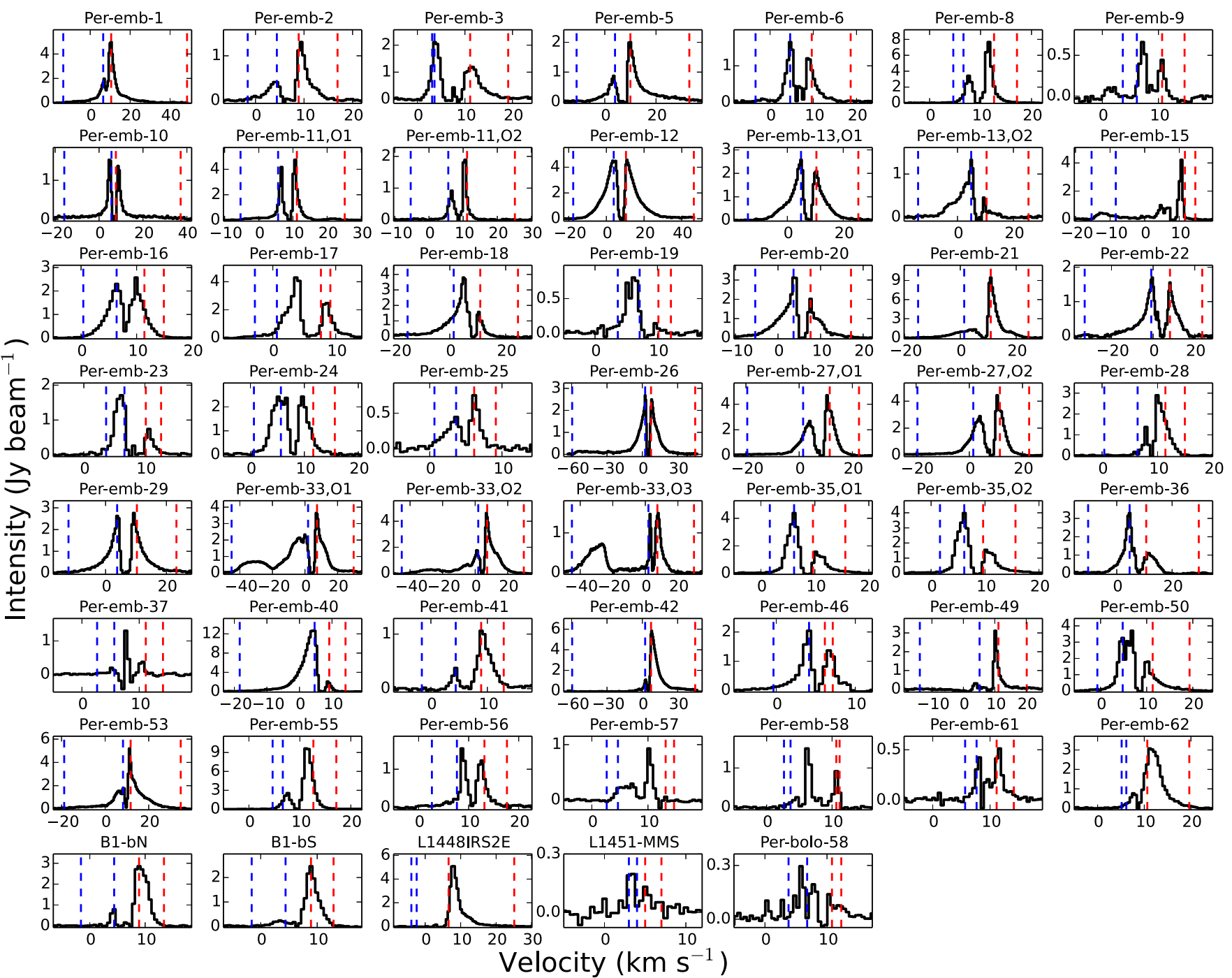

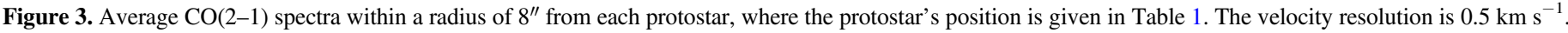

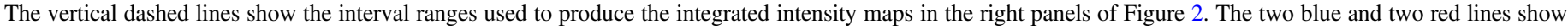

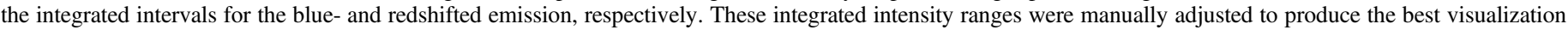

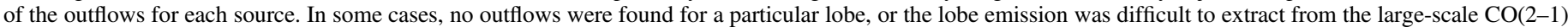

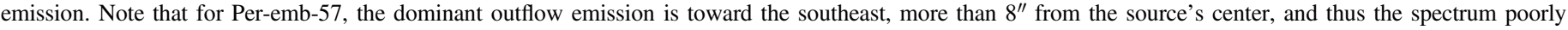
represents the outflow emission.

implemented in PYTHON. FILFINDER is unique in that it can find filaments with relatively low surface brightness compared to the main filaments, which is achieved by using an arctangent transform on the image. This algorithm first isolates the filamentary structure across the entire map. Then, each filament within the filamentary structure is made into a one-pixel-wide skeleton via the Medial Axis Transform (Blum 1967). We use the default implemented parameters in the FILFINDER algorithm, with the exception of the parameters size_thresh and skel_thresh, which were altered to provide the best visual fit to the actual Perseus data. Specifically, for these parameters we used the values size_thresh $=300$ and skel_thresh $=100$. The resolution of the observations $\left(36^{\prime \prime}\right)$ and the distance to the Perseus molecular cloud (235 pc) were also provided to the FILFINDER algorithm.

FILFINDER determines the filament direction via the Rolling Hough Transform (Clark et al. 2014). Unfortunately, the Rolling Hough Transform often performs poorly in the Perseus molecular cloud since FILFINDER sometimes combines distinct molecular clumps as a single filamentary structure. For example, FILFINDER combines NGC1333 and L1455 into a single filamentary network and measures the direction of the combined structure. We find that in most of these instances, the Rolling Hough Transform poorly estimates both the small- and large-scale filamentary direction. Instead of this transform, we approximate the filamentary direction by fitting a line to the filamentary skeleton output from FILFINDER. To do this, we first find the closest FILFINDER skeleton pixel to the position of the protostar given by Tobin et al. (2016). We then extract a square skeleton map of $11 \times 11$ pixels $\left(198^{\prime \prime} \times 198^{\prime \prime}\right.$ or $\sim 0.2 \mathrm{pc} \times$ $0.2 \mathrm{pc})$ centered on this closest skeleton pixel and fit an ordinary least squares bisector line (Isobe et al. 1990; Feigelson \& Babu 1992) to the scatter plot of the skeleton pixels. The slope of this fitted line is then converted to a PA. We use an extraction of an $11 \times 11$ pixel square because we find it large enough to fit the elongation of the filament, but small enough that the filament's direction is not strongly 

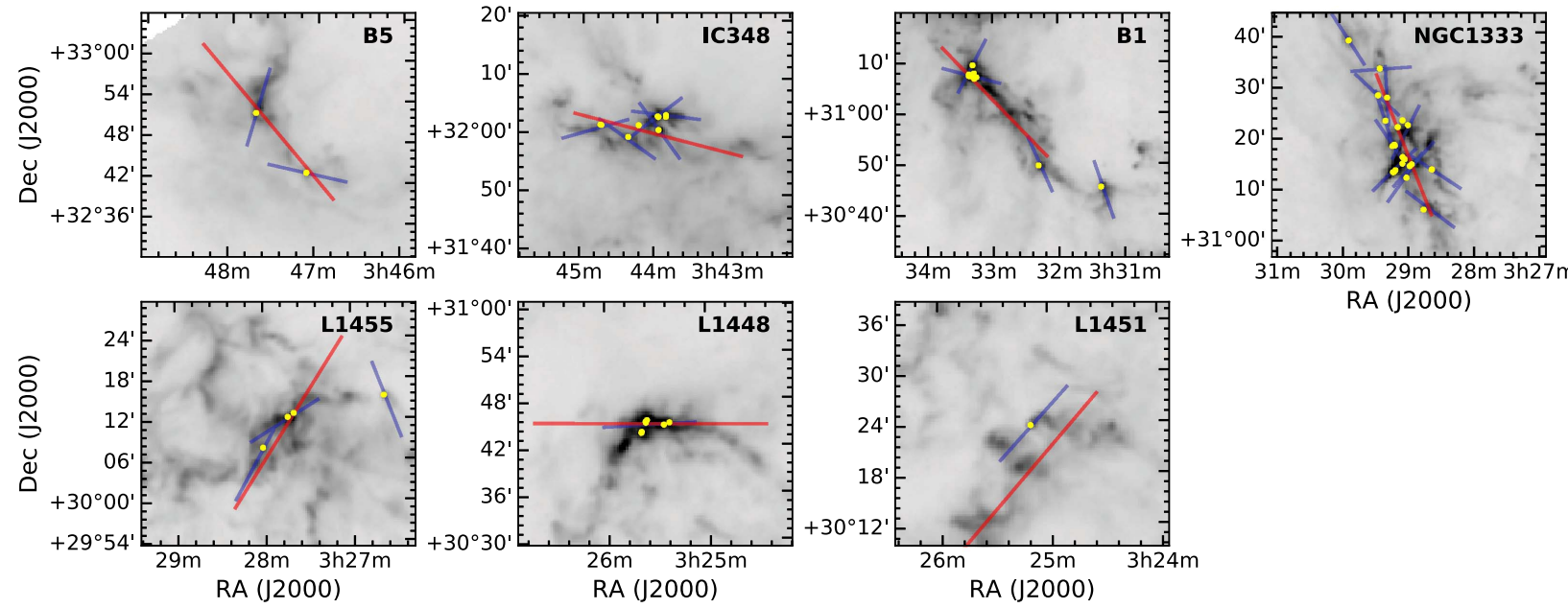

RA (J2000)

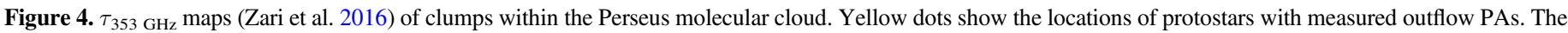

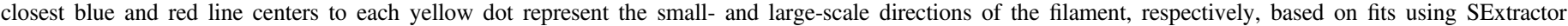

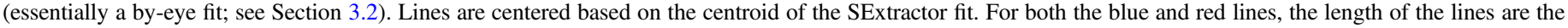
same angular size in each panel.

influenced by other nearby filamentary structures. We have also ran the same algorithm for extracting squares of skeleton pixels that are up to $\sim 3$ times larger or smaller than $11 \times 11$ pixels, and the results in our paper are qualitatively the same. The $11 \times 11$ pixel extraction provides the best visual fits to the filaments across all sources.

Figure 2 shows examples of this fitting process for two sources; other sources can be found in the figure set. Note that the measured filament PAs (red line in middle panels of Figure 2) are slightly off as one may measure by eye simply because nearby filament branches in the $11 \times 11$ pixel cutout of the skeleton map affects the bisector fit. In the rest of the paper, we will refer to this method for extracting filament directions as the "FILFINDER algorithm." In Table 2, we provide these filament angles, $\mathrm{PA}_{\mathrm{Fil}, \mathrm{F}}$, along with their corresponding projected outflow-filament angle, $\gamma_{\mathrm{F}}$.

Angular momentum of a protostar could possibly be inherited from filamentary structures larger than the filaments measured with $36^{\prime \prime}$ resolution. Therefore, we also make a comparison to larger scales by Gaussian smoothing the Zari et al. (2016) $\tau_{353 \mathrm{GHz}}$ maps and rerunning the FILFINDER algorithm discussed above. Specifically, we smooth the data to resolutions of $1^{\prime}, 2^{\prime}, 3^{\prime}, 4^{\prime}, 5^{\prime}$, and $6^{\prime}$, where $1^{\prime}$ is $0.068 \mathrm{pc}$, assuming a distance of $235 \mathrm{pc}$ to Perseus. FILFINDER progressively finds fewer branches in the Perseus filaments when we smooth $\tau_{353 \mathrm{GHz}}$ maps to these coarser resolutions. The measured projected outflow-filament angles for these resolutions are shown in Table 2 as $\gamma_{X^{\prime}}$, where $X^{\prime}$ is the smoothed resolution in arcminutes.

\subsubsection{Using SExtractor for Filament PAs}

The second method fits ellipses to the filaments via SExtractor (Bertin \& Arnouts 1996), as implemented in the Graphical Astronomy and Image Analysis Tool. ${ }^{17}$ SExtractor works by fitting ellipses to the emission data. We then adopt the PA of the fitted ellipses as the filament PA. To measure both the large- and small-scale filamentary structure, we extract two different filament directions for each protostar. For the large-scale structure, we fit a single filamentary direction to the clump

\footnotetext{
${ }^{17}$ https://www.dur.ac.uk/ pdraper/gaia/gaia.html
}

(i.e., the parsec-scale cloud structure), and for the small scale, we fit the most localized elongated structure for the protostar. For both scales, the parameters Detection threshold, Analysis threshold, and Contrast parameter were adjusted for each source so that the fitted ellipse best matches the elongation as judged by the human eye. We find that no single set of values for these three parameters can fit all filaments in the Perseus cloud that is agreeable with the human eye, and thus the parameters were adjusted filament-by-filament. Therefore, this method is primarily a "by eye" determination of the filament direction with the aid of software. This method of determining the filament PA is very similar to the method used in Anathpindika \& Whitworth (2008). We note that even at the small scale, the best SExtractor fit for a local filament may be the same for multiple protostars.

Figure 4 shows both the small- and large-scale filament PAs determined for each protostar using this method. The final projected outflow-filament angles using this method for both the small scale $\left(\gamma_{\mathrm{se}, \mathrm{S}}\right)$ and large scale $\left(\gamma_{\mathrm{se}, \mathrm{L}}\right)$ are given in Table 2. The measured filament PAs for both of these methods can be derived from $\gamma_{\mathrm{se}, \mathrm{S}}$ and $\gamma_{\mathrm{se}, \mathrm{L}}$ by using Equation (2) and the individual $\mathrm{PA}_{\mathrm{Out}}$ measurements.

\subsubsection{Comparison of the FILFINDER and SExtractor Techniques}

Both the FILFINDER and SExtractor filament-finding methods have their advantages and disadvantages. For example, the first method is completely automated, and if there are multiple filamentary branches in the field, the algorithm attempts to find the best filamentary direction in a fixed area of $\sim 0.2 \mathrm{pc} \times 0.2 \mathrm{pc}$. However, filamentary branches may be considered as a contaminate, in which case the second method (the SExtractor by-eye measurement) may more accurately determine the filamentary direction.

When comparing the two methods, the filament direction found with the FILFINDER algorithm are most comparable to those found at a small scale with SExtractor since these both measure filaments at approximately the same size scales. Figure 5 shows the absolute value of the difference in the measured angles $\gamma_{\mathrm{F}}$ and $\gamma_{\mathrm{se}, \mathrm{S}}$ for each protostar. Since PA $\mathrm{Put}_{\mathrm{Out}}$ for each protostar is measured the same regardless of the 


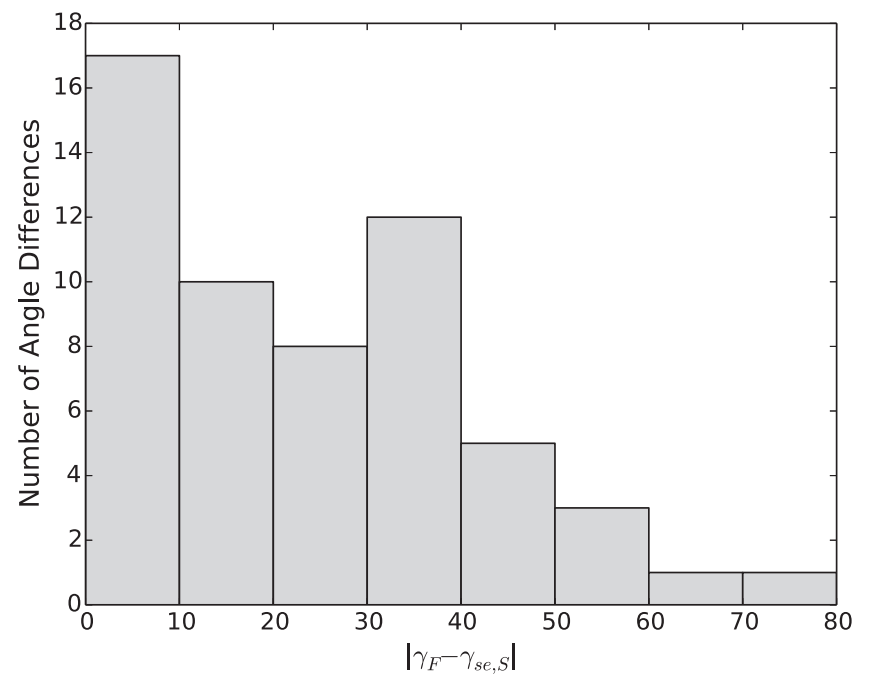

Figure 5. Histogram showing the magnitude of the difference in the projected outflow-filament angles measured by the two methods used to find the filament orientation. $\gamma$ values for the FILFINDER algorithm and the small-scale SExtractor fits are indicated by $\gamma_{\mathrm{F}}$ and $\gamma_{\mathrm{se}, \mathrm{S}}$, respectively.

method used to measure the filament orientation, $\gamma_{\mathrm{F}}-\gamma_{\mathrm{se}, \mathrm{S}}$ is equivalent to the difference in the measured filament directions for each technique. This histogram shows that the measured filament PAs mostly agree, but in some cases, the measured filament angles for each technique vary significantly. Therefore, in the following section, we present statistical comparisons to the outflows for both filamentfinding techniques.

\section{Results}

In this section, we analyze the distributions of $\mathrm{PA}_{\text {Out }}$ and $\gamma$. We note that, when we compare our empirical distributions of angles $\mathrm{PA}_{\mathrm{Out}}$ and $\gamma$ to simulated data, we favor the AndersonDarling (AD) test (e.g., Stephens 1974) over the KolmogorovSmirnov test. The AD test tends to be more powerful in detecting differences in distributions than the KolmogorovSmirnov test, particularly at the tail ends of the distributions (e.g., Hou et al. 2009; Engmann \& Cousineau 2011; Razali \& Wah 2011). While the $p$-values differ for these two tests, the overall statistical significance does not change dramatically and our conclusions remain unchanged. For the two-sample AD test, $p$-values near 1 imply that the two distributions are likely drawn from the same distribution, while $p$-values near 0 imply that they are unlikely drawn from the same distribution.

\subsection{Outflow Directions in Perseus}

Figure 6 shows a stacked histogram of $\mathrm{PA}_{\text {Out }}$, where the color of each stacked bar indicates the protostar's parental clump. As with Figure 1, this figure does not show any obvious relationship between $\mathrm{PA}_{\mathrm{Out}}$ and the protostar's parental clump. Since a stellar companion could possibly affect the spin axis of a protostar (e.g., Offner et al. 2016; Lee et al. 2017), we also show a stacked histogram of the "single" systems identified in the VANDAM survey (Tobin et al. 2016) in Figure 7. This survey used multi-wavelength data with resolutions as high as $15 \mathrm{au}$, and defined a system as a "single" system if it has no detected companions within 10,000 au. Again, the distribution is mostly random. We compare the "all" and "single system"

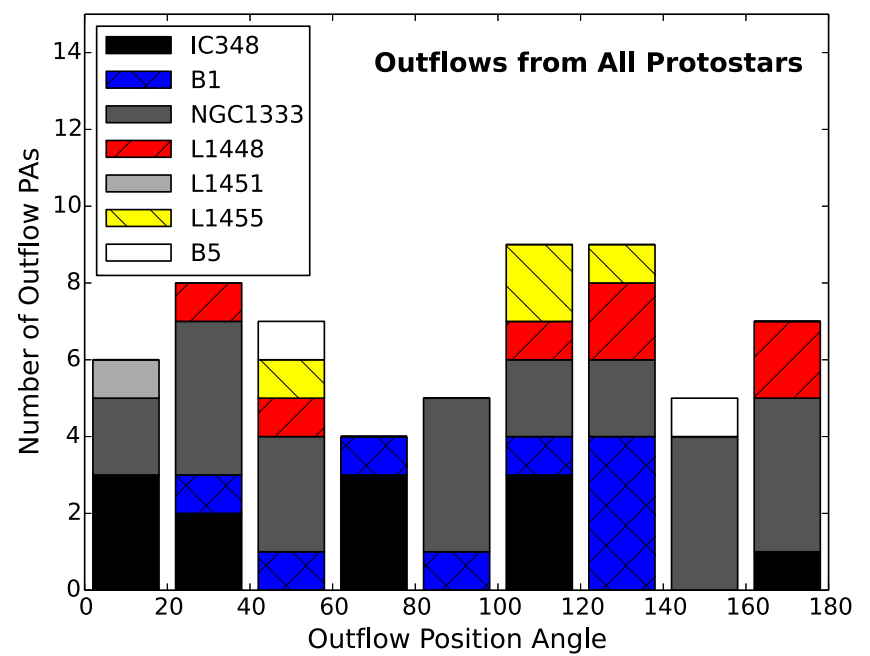

Figure 6. Stacked histogram (with $20^{\circ}$ bins) of outflow PAs in the Perseus molecular cloud. Colors correspond to the clump that $\mathrm{PA}_{\text {Out }}$ belongs to.

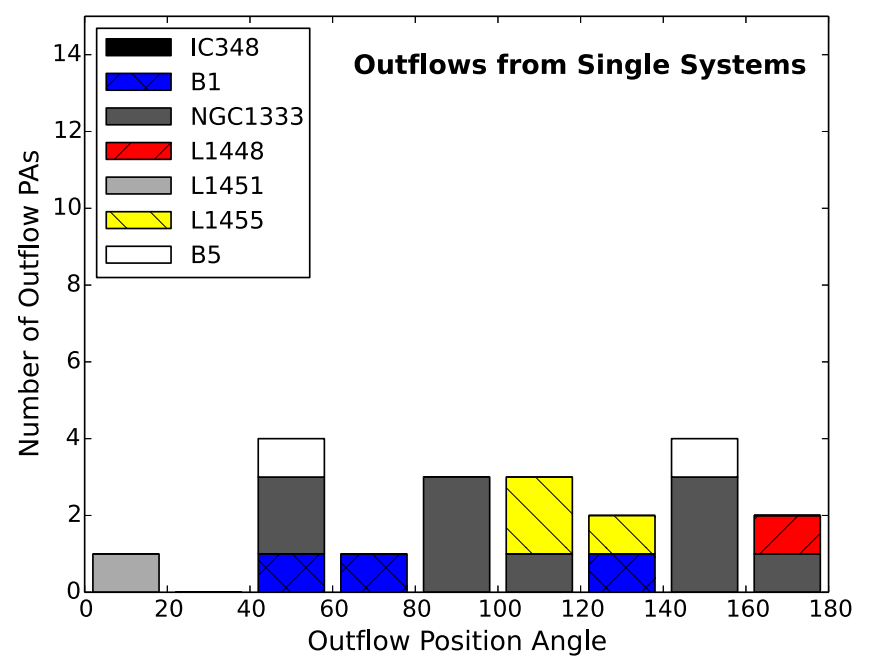

Figure 7. Same as Figure 6 but now only considering protostars that were not identified as multiples in the VANDAM survey (Tobin et al. 2016).

data to a random distribution, and the $\mathrm{AD}$ test gives $p$-values of 0.65 and 0.62 , respectively. This signifies that we cannot distinguish the $\mathrm{PA}_{\mathrm{Out}}$ histograms in Figures 6 and 7 from a random distribution of angles.

\subsection{Cumulative Distribution Functions (CDFs) Using FILFINDER Filament Angles}

While the first visual and clump regional tests did not show any obvious relationship between clump structure and protostellar outflow directions, clumps are parsec-sized, while filaments are about $0.1 \mathrm{pc}$ in diameter (e.g., Arzoumanian et al. 2011). As discussed in Section 3.2, we use FILFINDER to extract filament directions at the $36^{\prime \prime}(0.04 \mathrm{pc})$ scale. These filament directions, $\mathrm{PA}_{\mathrm{Fil}, \mathrm{F}}$, are then compared to $\mathrm{PA}_{\mathrm{Out}}$ to determine the projected outflow and filament angular difference, $\gamma_{\mathrm{F}}$. We plot the CDF of the observed $\gamma_{\mathrm{F}}$ in Figure 8. To investigate whether the distribution of $\gamma_{F}$ reflects outflows and filaments that are primarily aligned parallel, perpendicular, or at random, we perform 3D Monte Carlo simulations that we project onto 2D. Specifically, we simulate the CDF of the 


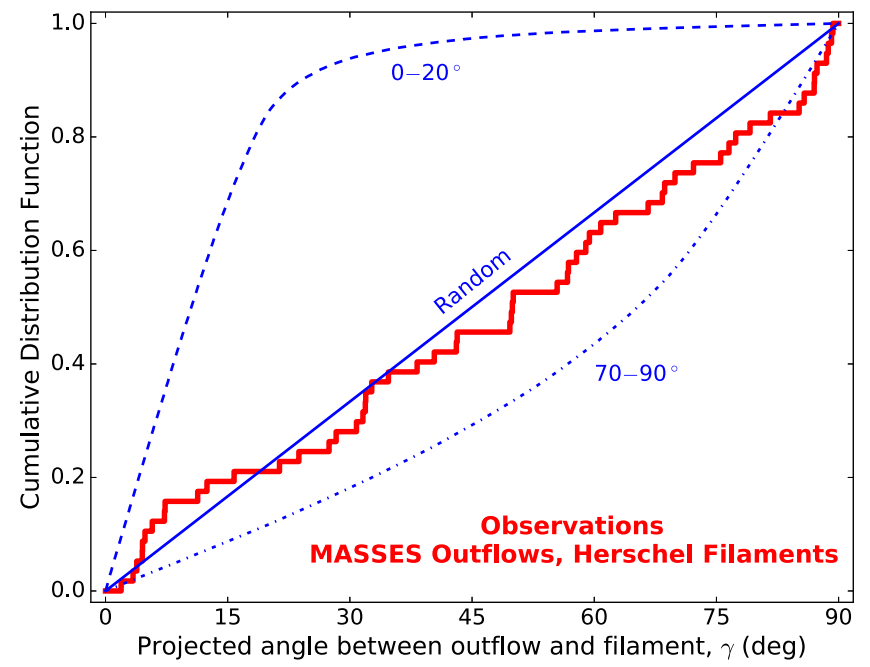

Figure 8. Cumulative distribution function of the projected angles between outflows and filaments, $\gamma$. The red step function shows $\gamma_{\mathrm{F}}$ for this study, which measures the angle between MASSES outflows and fitted Herschel filaments directions using the FILFINDER algorithm discussed in Section 3.2. The three blue lines show Monte Carlo simulations of the expected projected $\gamma$ for outflows and filaments that are three-dimensionally only parallel (actual outflow-filament angle that is between $0^{\circ}$ and $\left.20^{\circ}\right)$, only perpendicular $\left(70^{\circ}\right.$ $\left.90^{\circ}\right)$, or completely random $\left(0^{\circ}-90^{\circ}\right)$.

expected projected angles in the sky for outflow-filament angles that are three-dimensionally "only parallel" (defined as actual outflow-filament angles that are distributed between $0^{\circ}$ and $20^{\circ}$ ), "only perpendicular" (actual angles between $70^{\circ}$ and $90^{\circ}$ ), or completely random (actual angles between $0^{\circ}$ and $90^{\circ}$ ). The expected observed (i.e., projected) $\gamma$ for these three Monte Carlo instances are also shown in Figure 8. Detailed information on the Monte Carlo simulations is presented in Appendix A.

Immediately evident from Figure 8 is that the distribution of $\gamma_{F}$ is inconsistent with outflows and filaments that are preferentially parallel. The projected angles are also inconsistent with a purely perpendicular alignment with over $99 \%$ confidence (the AD test gives a $p$-value $=0.0045$ ). However, we cannot significantly distinguish the $\gamma_{F}$ distribution from a distribution of randomly aligned outflows and filaments $(p$-value $=0.20)$. Table 3 summarizes the statistical tests conducted on all the $\gamma$ measurements discussed in Section 3. In Figure 9, we show the distribution of $\gamma_{\mathrm{F}}$ as a stacked histogram, with colors representing the parental clump. No obvious nonrandom relationship is found, regardless of the protostar's clump location.

So far, we produced simple models of $\gamma$ from outflowfilament angles that are only parallel, only perpendicular, or aligned at random. As mentioned in Section 1, outflow orientation may be determined by the dominant flow direction about the filament. Therefore, a bimodal distribution of $\gamma$ is possible, e.g., a mix of both parallel and perpendicular orientations.

We test different three-dimenisonal combinations of purely parallel (again, where angles are distributed between $0^{\circ}$ and $20^{\circ}$ ) and purely perpendicular (angles between $70^{\circ}$ and $90^{\circ}$ ) outflow-filament angles via Monte Carlo simulations. We consider 101 bimodal cases in increments of $1 \%$ (i.e., $100 \%$ parallel, $99 \%$ parallel and $1 \%$ perpendicular, $98 \%$ parallel and

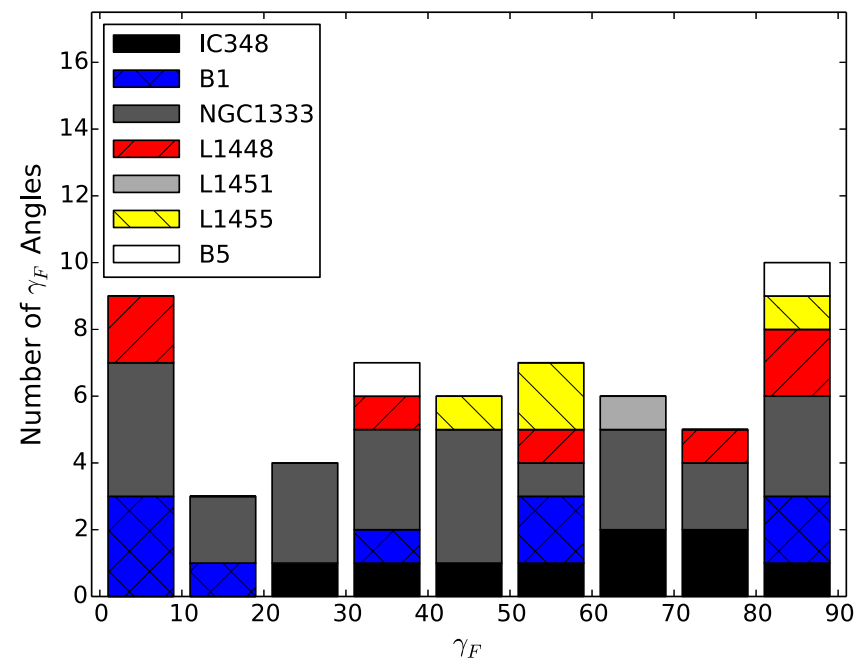

Figure 9. Same as Figure 6, but now the stacked histogram is shown for $\gamma_{\mathrm{F}}$. The histogram bin size is $10^{\circ}$.

Table 3

Anderson-Darling Test $p$-values

\begin{tabular}{lcl}
\hline \hline $\begin{array}{l}\text { Empirical } \gamma \\
\text { Distribution }\end{array}$ & $\begin{array}{c}p \text {-value, Compared } \\
\text { with Random }\end{array}$ & $\begin{array}{l}p \text {-value, Compared } \\
\text { with Perpendicular }\end{array}$ \\
\hline$\gamma_{\mathrm{F}}$ & 0.20 & 0.0045 \\
$\gamma_{1^{\prime}}$ & 0.33 & 0.00085 \\
$\gamma_{2^{\prime}}$ & 0.40 & 0.0011 \\
$\gamma_{3^{\prime}}$ & 0.42 & 0.0029 \\
$\gamma_{4^{\prime}}$ & 0.49 & 0.00024 \\
$\gamma_{5^{\prime}}$ & 0.24 & 0.023 \\
$\gamma_{6^{\prime}}$ & 0.59 & 0.00069 \\
$\gamma_{\mathrm{se}, \mathrm{S}}$ & 0.74 & 0.00014 \\
$\gamma_{\mathrm{se}, \mathrm{L}}$ & 0.64 & 0.0021 \\
Anathpindika \& Whitworth & 0.017 & 0.16 \\
$\gamma_{\mathrm{F}}$, Single Protostars & 0.60 & 0.18 \\
$\gamma_{\mathrm{F}}$, Multiple Protostars & 0.20 & 0.011 \\
$\gamma_{\mathrm{F}}$, with $T_{\text {bol }}<50$ & 0.53 & 0.021 \\
$\gamma_{\mathrm{F}}$, with $T_{\text {bol }}>50$ & 0.18 & 0.075 \\
$\gamma_{\mathrm{F}}$, with $\tau_{353 \mathrm{GHz}}<0.016$ & 0.15 & 0.20 \\
$\gamma_{\mathrm{F}}$, with $\tau_{353 \mathrm{GHz}}>0.016$ & 0.27 & 0.0050 \\
\hline
\end{tabular}

Note. $p$-values are not shown for empirical $\gamma$ distributions compared with parallel $\gamma$ distributions because they are all extremely low in value (less than $10^{-9}$ ).

$2 \%$ perpendicular, ..., $100 \%$ perpendicular). Figure 10 shows the CDFs of several of these bimodal distributions projected into two dimensions. We find that, when comparing to the observed distribution $\gamma_{\mathrm{F}}$, the simulated $\gamma$ that is a mix of $22 \%$ parallel and $78 \%$ perpendicular maximizes the $p$-values for the AD test (as well as the Kolmogorov-Smirnov test). The $p$-value for this case is 0.55 , signifying a slightly more consistent distribution with the observed $\gamma_{\mathrm{F}}$ distribution than a random distribution.

This bimodal test can also constrain which mixes of parallel and perpendicular are unlikely. According to the AD test, we find that at $95 \%$ confidence, $\gamma_{\mathrm{F}}$ probably does not come from a bimodal distribution that is more than $39 \%$ parallel or more than $94 \%$ perpendicular. At $85 \%$ confidence, we find that the 


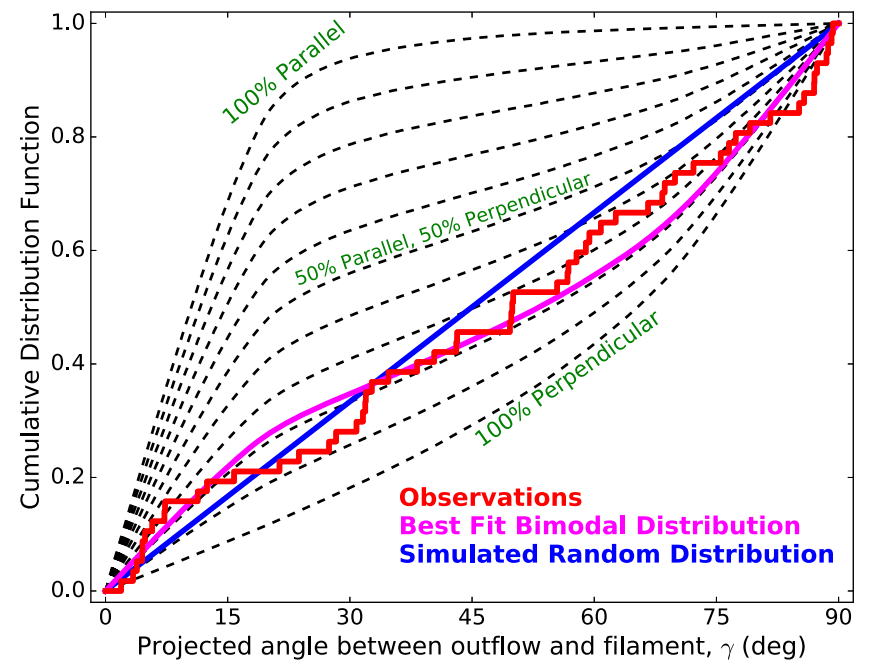

Figure 10. Cumulative distribution function of the projected angles between outflows and filaments, $\gamma$, with the red step curve showing the empirical distribution, $\gamma_{\mathrm{F}}$. Black dashed lines show different mixes of projected outflowfilament angles that are three-dimensionally parallel and perpendicular in increments of $10 \%$ (i.e., the top line is $100 \%$ parallel and $0 \%$ perpendicular, the next line is $90 \%$ parallel and $10 \%$ perpendicular, and so on). Parallel angles are defined as three-dimensional angles drawn from a distribution between $0^{\circ}$ and $20^{\circ}$, while perpendicular angles are defined as angles drawn from a distribution between $70^{\circ}$ and $90^{\circ}$ (see Appendix A for details). The blue line shows a random distribution of projected angles, while the magenta line shows the best bimodal fit to the data of $22 \%$ parallel and $78 \%$ perpendicular.

$\gamma_{\mathrm{F}}$ distribution does not come from a bimodal distribution that is more than $33 \%$ parallel or more than $90 \%$ perpendicular.

Other mixes of $\gamma$ distributions are also possible, such as mixes of a random distribution with perpendicular and/or parallel distributions. We do not test other distribution mixes in this paper since we mainly want to show that perpendicular outflows and filaments are much more likely than parallel.

As discussed in Section 3.2, we also determine filament angles by running the FILFINDER algorithm on Perseus $\tau_{353 \mathrm{GHz}}$ maps that have been smoothed to coarser resolution. The resulting CDFs for $\gamma$ at these resolutions are shown in Figure 11. We find that the CDFs at all resolutions are similar with each other, with the AD test $p$-value 0.45 or greater when comparing any two distributions. We also find consistent results between the smoothed and the non-smoothed $\left(36^{\prime \prime}\right)$ resolution $\gamma$ angles. Specifically, as shown in Table 3, none of the $\gamma$ distributions extracted from the smoothed $\tau_{353 \mathrm{GHz}}$ maps can be statistically distinguished from a random distribution, but all are inconsistent with projected angles from only perpendicular and only parallel distributions.

\subsection{CDFs Using SExtractor Filament Angles}

In Figure 12, we show the CDFs when using the SExtractor filament direction fits, which is essentially a fit by eye (see Section 3.2). We find similar results for both the small-scale (i.e., fitting the closest elongated feature to each protostar) and large-scale (i.e., fitting the main part of the clump containing each group of protostars) SExtractor fitting as with the FILFINDER algorithm. That is, the SExtractor fits are not inconsistent with a random distribution and are significantly inconsistent with both parallel and perpendicular angle distributions (see Table 3). Also shown in this figure are the

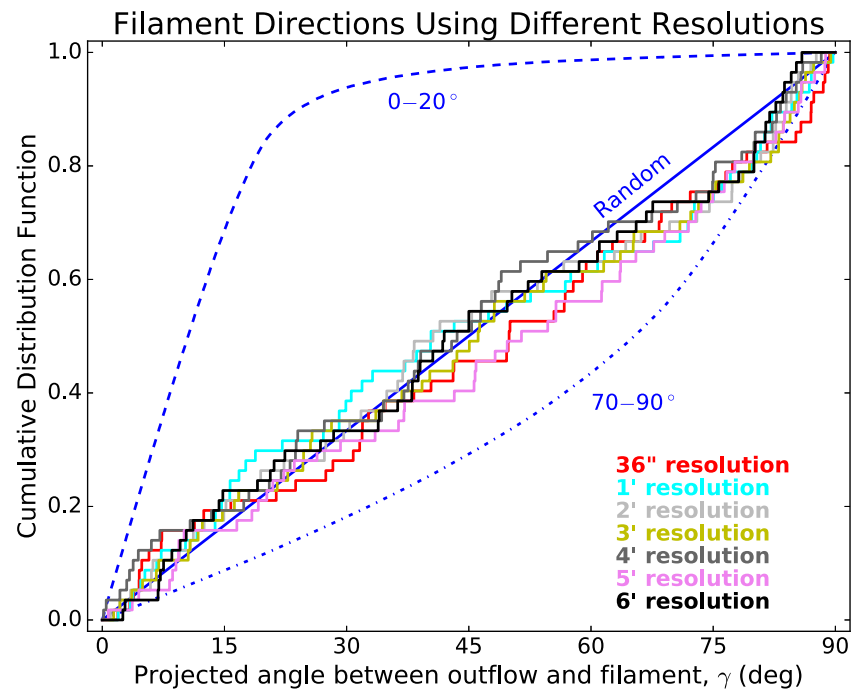

Figure 11. Same as Figure 8, except with additional step curves showing the effects of smoothing the Perseus $\tau_{353 \mathrm{GHz}}$ map before running the FILFINDER algorithm described in Section 3.2. The colors indicate which resolution the $\tau_{353 \mathrm{GHz}}$ map was smoothed to in creating the empirical $\gamma \mathrm{CDF}$.

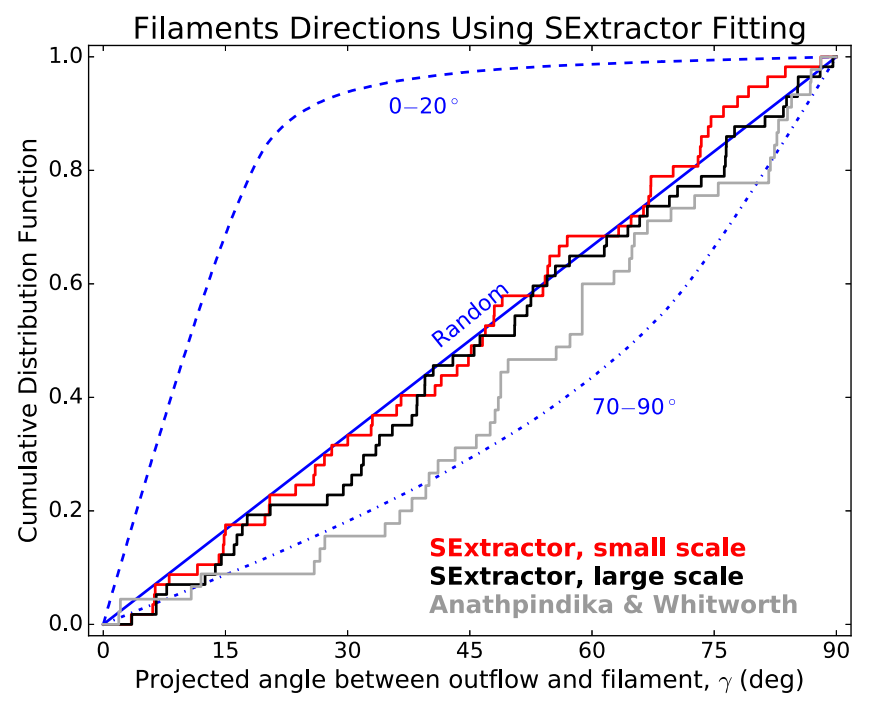

Figure 12. Figure caption is the same as Figure 8, except now the step functions show the SExtractor fitting of filament directions (which is essentially a "by eye" fit) at both small- and large scales, as described in Section 3.2. The results from Anathpindika \& Whitworth (2008) are also shown, but we caution any interpretation of this curve due to shortcomings of the study discussed in Appendix B.

results from Anathpindika \& Whitworth (2008), who use a similar filament fitting algorithm. Unlike our results, their distribution for $\gamma$ is more consistent with perpendicular ( $p$-value of 0.17 ) than random ( $p$-value of 0.017). However, we caution an interpretation of the Anathpindika \& Whitworth (2008) $\gamma$ distribution due to several shortcomings in their study, which are discussed in detail in Appendix B. We do not show the results from Davis et al. (2009) because they do not supply any information on $\gamma$ or the filament PAs.

As in Section 4.2, we also test which bimodal distribution of parallel and perpendicular projected orientations matches the 

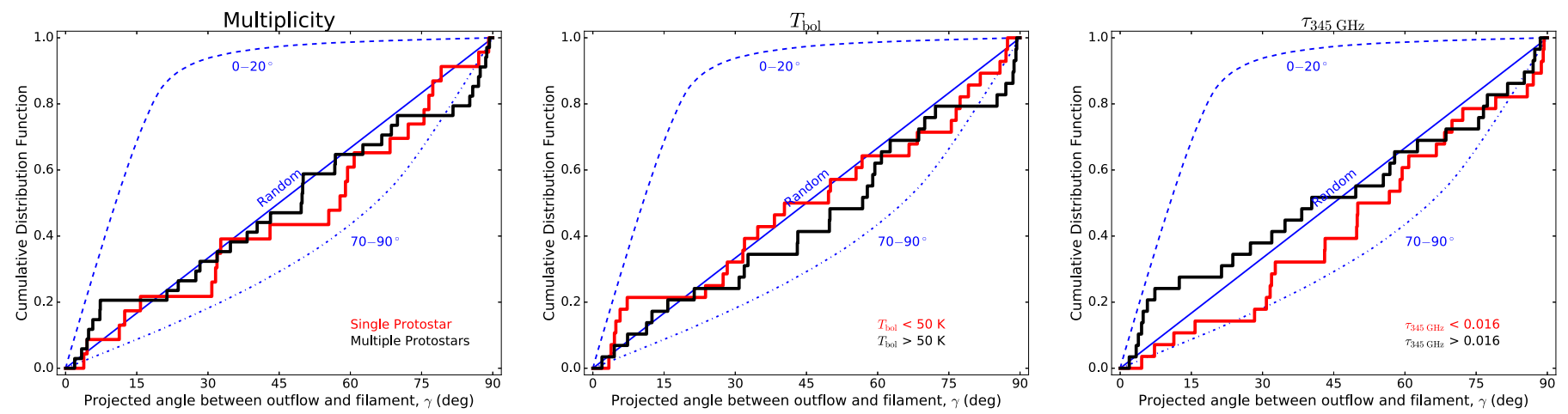

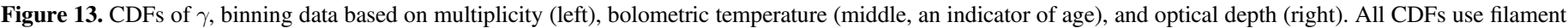
measurements from the FILFINDER algorithm.

observations using SExtractor filament fits. The results are similar as those found with FILFINDER.

\subsection{CDFs Based on Protostellar Characteristics}

Here we investigate whether or not individual characteristics of the protostars themselves or their surrounding environment affect the underlying $\gamma$ distribution. We consider the protostar's multiplicity, the protostar's bolometric temperature $\left(T_{\text {bol }}\right)$, and the Zari et al. (2016) $\tau_{353 \mathrm{GHz}}$ pixel value at the protostar (see Table 1 for these values). Both the protostellar multiplicity and $T_{\text {bol }}$ were taken from Tobin et al. (2016) and references therein. For multiples that were resolved with the VLA but not with Spitzer, we assign the same $T_{\text {bol }}$ for all multiples within the Spitzer-defined source. The left panel of Figure 13 shows two CDFs: one for systems that have only one known protostar within 10,000 au and another for systems with more than one known protostar within 10,000 au. The middle panel shows two CDFs based for protostars with $T_{\text {bol }}$ above and below $50 \mathrm{~K}$, where lower $T_{\text {bol }}$ indicates younger protostars. The right panel shows two CDFs based on the $\tau_{353 \mathrm{GHz}}$ pixel value at the Tobin et al. (2016) protostellar location for $\tau_{353 \mathrm{GHz}}$ above and below 0.016 . Protostars at locations of higher $\tau_{353 \mathrm{GHz}}$ are more likely to be in their natal star-forming filament. We select delimitations of $T_{\mathrm{bol}}=50 \mathrm{~K}$ and $\tau_{353 \mathrm{GHz}}=0.016$ so that roughly half of the sample is in each CDF. We note that $T_{\text {bol }}=70 \mathrm{~K}$ is typically used to separate Class 0 and Class I protostars (Chen et al. 1995; Enoch et al. 2009).

Since the distribution of $\gamma_{\mathrm{F}}$ angles is separated into two CDFs for each panel in Figure 13, statistically differentiating the distributions from random and perpendicular Monte Carlo simulations is more difficult. Table 3 shows that none of these CDFs can be distinguished from a random distribution, and several CDFs are statistically inconsistent with perpendicular. While we only show the corresponding $p$-value results if we use the FILFINDER algorithm, the results would be qualitatively the same if we used the filament fits from SExtractor.

We also find that the empirical CDFs in each panel are not inconsistent with each other. Specifically, the $p$-value between singles and multiples is 0.80 , between the two $T_{\text {bol }}$ bins is 0.56 , and between the two $\tau_{353 \mathrm{GHz}}$ bins is 0.24 . The latter shows that $\tau_{353 \mathrm{GHz}}$ could possibly be the best discriminator between two populations of $\gamma$. This would imply that protostars that are less embedded (and likely older) have outflows perpendicular to their natal filaments. Indeed, this idea is supported by the fact that higher $T_{\text {bol }}$ (i.e., older protostars) are closer to the perpendicular curve (albeit, very slightly) than sources with lower $T_{\text {bol }}$. However, we stress that this trend is only tentative because it is far from being statistically significant to draw firm conclusions. A much larger sample of protostars would allow for a better understanding of whether or not individual protostellar characteristics affect the observed $\gamma$ distribution.

\section{Discussion}

We find that the observed distribution of the projected angle between outflow and filaments, $\gamma$, is significantly inconsistent with projected "only parallel" (angles between $0^{\circ}$ and $20^{\circ}$ ) and "only perpendicular" (angles between $70^{\circ}$ and $90^{\circ}$ ) angle distributions. The observed $\gamma$ distribution instead appears more consistent with a random distribution and for certain bimodal distributions of parallel and perpendicular angles. The best match for the bimodal distribution are angles that are only parallel $22 \%$ of the time and only perpendicular $78 \%$ of the time. These results are at apparent disagreement with Anathpindika \& Whitworth (2008), but that study has a number of caveats, as explained in Appendix B. Therefore, we believe that, at least in Perseus, our results are a better representation of the actual $\gamma$ distribution.

Davis et al. (2009) also found an apparently random alignment when comparing molecular hydrogen outflows to the filament/core directions in Orion, but they did not test the idea of a mixed distribution of only parallel and only perpendicular angles. Such random alignment is supported by Tatematsu et al. (2016), who found that the angular momentum axes of cores in the Orion A filament are random with respect to the filamentary structure. Our study and these studies show that protostellar outflows in both low- and high-mass starforming regions show no preferred orientation relative to their local filament. In a study that does not compare outflow angles to filaments, Ioannidis \& Froebrich (2012) investigated whether outflows are perpendicular to the Galactic plane. Specifically, they observed molecular hydrogen outflows within part of the Galactic plane $\left(18^{\circ}<l<30^{\circ} ;-1.5<b<+1.5\right)$, and they also found a somewhat random distribution of outflow PAs, with a marginal preference for outflows to be aligned perpendicular to the Galactic plane.

Theoretical models and simulations at parsec-scales have shown that filaments can be the result of colliding clouds or 
flows, and the initial orientation of the angular momentum in these systems can dictate how angular momentum is transported to smaller scales. Theoretical expectations of $\gamma$ vary significantly and can often depend on the initial conditions set in the simulation. Hydrodynamic turbulent simulations of collapsing clouds by Tilley \& Pudritz (2004) show that cores within filaments can form at oblique shocks, and these shocks can impart angular momentum to the core. Simulations by Clarke et al. (2017) show that filaments accreting from a turbulent medium have a vorticity (and hence, angular momentum) that is typically parallel to filaments, which is primarily derived from radial inhomogeneous accretion. Chen \& Ostriker $(2014,2015)$ included magnetohydrodynamics in their simulations and found that for filaments forming due to converging flows, mass flows along magnetic field lines to both the filaments and cores (which form simultaneously). For dense filaments of size scales on the order of $0.1 \mathrm{pc}$, some observations have suggested that magnetic field lines are perpendicular to the filament's elongation (e.g., Matthews \& Wilson 2000; Pereyra \& Magalhães 2004; Santos et al. 2016). If such fields help drive gas perpendicular to the filaments, the results from Clarke et al. (2017) suggest that this could induce a vorticity parallel to the filaments. The ability for such vorticity to be transferred to angular momentum at the core scale or smaller is unclear, and this was not investigated by Clarke et al. (2017). However, if angular momentum is inherited by the protostar in the same direction of the vorticity, we would expect the rotation of the protostar to be parallel with the filament. Indeed, simulations by Tilley \& Pudritz (2004) and Banerjee et al. (2006) show that for filaments forming due to colliding flows, oblique shocks can impart net rotation parallel to the filament, which in turn can produce parallel filaments and protostellar rotation axes. However, numerical simulations by Whitworth et al. (1995) suggest that filaments can form via two colliding clumps, and the initial net angular momentum of the system will typically be perpendicular to the filaments that form. The protostar can inherent this angular momentum, and thus its rotation axis will tend to be perpendicular to the filament. Theoretical predictions of rotation axes either parallel or perpendicular to the filament axes are at odds with observations at both the core (Tatematsu et al. 2016) and protostellar scales (this study).

Since filaments may be created through a variety of mechanisms, a combination of these mechanisms could cause outflow-filament alignment to appear more randomly aligned. Assuming the alignment is not purely random, our observations suggest that outflows are more likely to form perpendicular than parallel to the filamentary elongation. Unfortunately, twodimensional projections of three-dimensionally random and mostly perpendicular distributions look quite similar, making it difficult for even large samples to distinguish between the two. Moreover, the fact that the angles between outflows and filaments are neither purely parallel nor purely perpendicular may reflect how material is funneled toward the protostars at both the large and small scales. On large scales, Chen \& Ostriker (2014) suggested that material flows along magnetic field lines, which could be mainly perpendicular to the filament along its exterior and parallel within the interior. This mix of flows could induce a more random-like vorticity to the parental cores of the protostars.
Higher resolution simulations have explored angular momentum transfer within cores (i.e., scales $\lesssim 0.1 \mathrm{pc}$ ). Walch et al. (2010) used smoothed particle hydrodynamic simulations of a low-mass, transonically turbulent core, and found that the rotation axes of protostars tend to be perpendicular to "small" filaments (diameters $\sim 0.01 \mathrm{pc}$ ) within cores. However, the Herschel-derived $\tau_{353 \mathrm{GHz}}$ maps $\left(36^{\prime \prime}=0.04 \mathrm{pc}\right.$ resolution) do not resolve these small filaments. Observations of molecular line (e.g., Hacar et al. 2013) or continuum tracers (e.g., Pineda et al. 2011b) suggest that filaments break into smaller substructures, and therefore the initial conditions for protostellar rotation and collapse may be set by these smaller structures. These substructures sometimes have similar elongation to their parent filaments (Pineda et al. 2011b; Hacar et al. 2013), but not always (e.g., Pineda et al. 2010, 2015). At scales of $\sim 10,000 \mathrm{au}$, elongated, flattened envelopes are observed to be perpendicular to their outflows (e.g., Looney et al. 2007). The typical size of these flattened structures and their universality remains unclear. Observational surveys that probe dense structures at scales between $\sim 0.01$ and $0.1 \mathrm{pc}$ can uncover whether and at what scale an elongated structure is perpendicular with a protostar's angular momentum axis.

Regardless of the initial conditions that create filaments, the actual spin of a protostar may be independent of the filamentary structure. The local vorticity of turbulence may determine the spin of the parent core (McKee \& Ostriker 2007). Even within the core, the rotation axes of protostars may change. Offner et al. (2016) and Lee et al. (2017) found that both turbulent accretion onto a protostar and interaction with companions can cause a significant evolution in a protostar's spin. Essentially, at small scales, it is feasible that the underlying structure, turbulence, and/or multiplicity could significantly alter the initial rotation axes. While random alignment is favored in some models of turbulent accretion, even models with strong magnetic fields could result in random alignment. Mouschovias \& Morton (1985) suggested that for fragments linked by strong magnetic fields, the angular momentum orientation of the fragments depends solely on the shape of the magnetic flux tubes, which can have quite irregular shapes. If fragments in filaments are indeed magnetically linked, our study suggests that the flux tubes connecting them are indeed irregular. Theoretical simulations have begun to incorporate gravity, turbulence, magnetic fields, and outflows to study the formation of filamentary complexes (e.g., Myers et al. 2014; Federrath 2016). Such simulations can supply a more robust expectation of the observed distribution of $\gamma$ for a large sample of outflows and filaments.

\section{Summary}

The MASSES survey observed $\mathrm{CO}(2-1)$ in all the known Class 0/I protostars in the Perseus molecular cloud. With these data, along with ancillary observations of $\mathrm{CO}$ rotational transitions, we were able to determine the outflow PAs for each protostar. We compare these angles to the filament directions based on optical depth maps derived from Herschel (Zari et al. 2016). We find the following.

1. The outflow directions are randomly distributed in the Perseus molecular cloud. This random distribution appears to hold regardless of the parental clump of a protostar. 
2. The projected angle between the outflow and filament, $\gamma$, is significantly inconsistent with a "purely parallel" and a "purely perpendicular" distribution of projected angles.

3. The observed $\gamma$ distribution cannot be distinguished from a random distribution.

4. We also consider bimodal distributions, and find a slightly more consistent distribution to the observed gamma distribution when $22 \%$ of the projected angles are parallel and $78 \%$ are perpendicular. Our observations are unlikely to come from bimodal distributions that are more than $\sim 33 \%$ parallel or more than $\sim 90 \%$ perpendicular.

5. Regardless of the multiplicity, $T_{\text {bol }}$ (age), or opacity of the individual protostars, the observed $\gamma$ distribution cannot be distinguished from a random distribution. However, to better test how these different parameters of the protostars affect the $\gamma$ distribution, a larger sample is needed.

We discuss the implications of the fact that outflows and filaments are neither purely perpendicular or purely parallel. We suggest that this feature could reflect the physical conditions at large or small scales. At large scale, a dominant flow direction toward cores may not exist. At small scale, the underlying structure, turbulence, and/or multiplicity could affect the angular momentum axes. Observational surveys of dust emission at scales between $\sim 0.01$ and $0.1 \mathrm{pc}$ are needed to reveal whether and how a protostar's angular momentum axis may be related to its natal structure.

We thank an anonymous referee for thorough and helpful reviews. I.W.S. acknowledges support from NASA grant NNX14AG96G. E.I.V. acknowledges support form the Russian Ministry of Education and Science grant 3.5602.2017. J.J.T. acknowledges support from the University of Oklahoma, the Homer L. Dodge endowed chair, and grant 639.041.439 from the Netherlands Organisation for Scientific Research (NWO). J.E.P. acknowledges the financial support of the European Research Council (ERC; project PALs 320620). The authors thank the SMA staff for executing these observations as part of the queue schedule, Charlie Qi and Mark Gurwell for their technical assistance with the SMA data, and Eric Keto for his guidance with SMA large-scale projects. The Submillimeter Array is a joint project between the Smithsonian Astrophysical Observatory and the Academia Sinica Institute of Astronomy and Astrophysics and is funded by the Smithsonian Institution and the Academia Sinica. This research has made use of the VizieR catalogue access tool and the SIMBAD database operated at CDS, Strasbourg, France. This research made use of APLpy, an open-source plotting package for Python (Robitaille \& Bressert 2012).

\section{Appendix A Monte Carlo simulations}

Many studies have used Monte Carlo simulations to show the expected observed distribution of angles of two vectors projected into three dimensions. Several of these studies (Hull et al. 2013, 2014; Lee et al. 2016; Offner et al. 2016) were specifically interested in the same projected distributions we are interested in this study, i.e., the projection of angles that are threedimensionally purely parallel (between $0^{\circ}$ and $20^{\circ}$ ), purely perpendicular $\left(70^{\circ}-90^{\circ}\right)$, or completely random $\left(0^{\circ}-90^{\circ}\right)$. These studies do not discuss the exact details of the Monte Carlo simulations. Here we discuss our Monte Carlo method, and the results are consistent with the aforementioned studies.

For our methodology, we generated $N$ pairs of threedimensional vectors with each vector random about the sky. To generate a random vector, we chose a random point on the surface of a unit sphere and then connected the sphere's origin to this point. For the purpose of Monte Carlo simulations, sampling a random point from a unit sphere that avoids biases has been well-studied (e.g., Marsaglia 1972). We outline one such way to select random points on a unit sphere below, which is based on Weisstein (2017). We first selected a random angle $\theta$ between 0 and $2 \pi$ and a random number $u$ that is between -1 and 1 . From random variables $\theta$ and $u$, we then selected a random point on a unit sphere at position $x, y$, and $z$, where

$$
\begin{gathered}
x=\sqrt{1-u^{2}} \cos \theta, \\
y=\sqrt{1-u^{2}} \sin \theta, \\
z=u .
\end{gathered}
$$

A unit vector between the sphere's origin and this point is

$$
\boldsymbol{v}=\left[\begin{array}{l}
x \\
y \\
z
\end{array}\right] .
$$

To randomly sample from all angles within a unit sphere, we generated two random unit vectors, $\boldsymbol{v}_{1}$ and $\boldsymbol{v}_{2}$, and measure the angle between the vectors. The angle is simply

$$
\gamma_{3 \mathrm{D}}=\arccos \left(\boldsymbol{v}_{1} \cdot \boldsymbol{v}_{2}\right) \text {. }
$$

Since we are interested in the smallest angle created by the two intersecting vectors, we constrained $\gamma_{3 \mathrm{D}}$ to be between $0^{\circ}$ and $90^{\circ}$, e.g., if $\gamma_{3 \mathrm{D}}$ is larger than $90^{\circ}$, we subtracted $\gamma_{3 \mathrm{D}}$ from $180^{\circ}$. We generated $N$ pairs of vectors to produce $N$ angles of $\gamma_{3 \mathrm{D}}$. For the Monte Carlo simulations in this paper, we chose $N=10^{6}$. We show the distribution of $\gamma_{3 \mathrm{D}}$ for $N=10^{6}$ via the histogram in Figure 14. We then mapped each $\gamma_{3 \mathrm{D}}$ angle to a projected angle in $2 \mathrm{D}, \gamma$, by setting one axis for the vector pair to 0 (the $x$-value of the vector in our code) and calculating the new angle between the vectors.

From this mapping, we can extract a range of angles from the distribution of $\gamma_{3 \mathrm{D}}$ and plot its corresponding $\gamma$ distribution. For this study, we were primarily interested in projections for threedimensional angles that are purely parallel (between $0^{\circ}$ and $\left.20^{\circ}\right)$, purely perpendicular $\left(70^{\circ}-90^{\circ}\right)$, or completely random $\left(0^{\circ}-90^{\circ}\right)$. For the Monte Carlo sample size of $N=10^{6}$ (equivalent to the number for the completely random sample size), we extracted from the $\gamma_{3 \mathrm{D}}$ distribution $\sim 60,000$ projections for a purely parallel sample and $\sim 340,000$ for a purely perpendicular sample. The reason why the sample size for purely perpendicular is much larger than purely parallel is simply due to the fact that perpendicular-like angles are much more likely for two random vectors in a unit sphere (Figure 14). Our tests show that the curve of the CDF of the Monte Carlo simulation (e.g., Figure 8 ) is very smooth as long as the sample size is larger than $\sim 20,000$ projections.

\section{Appendix B Discrepancy with Anathpindika \& Whitworth}

As seen in Table 3 and Figure 12, (Anathpindika \& Whitworth 2008, henceforth in this appendix, AW08) found a 


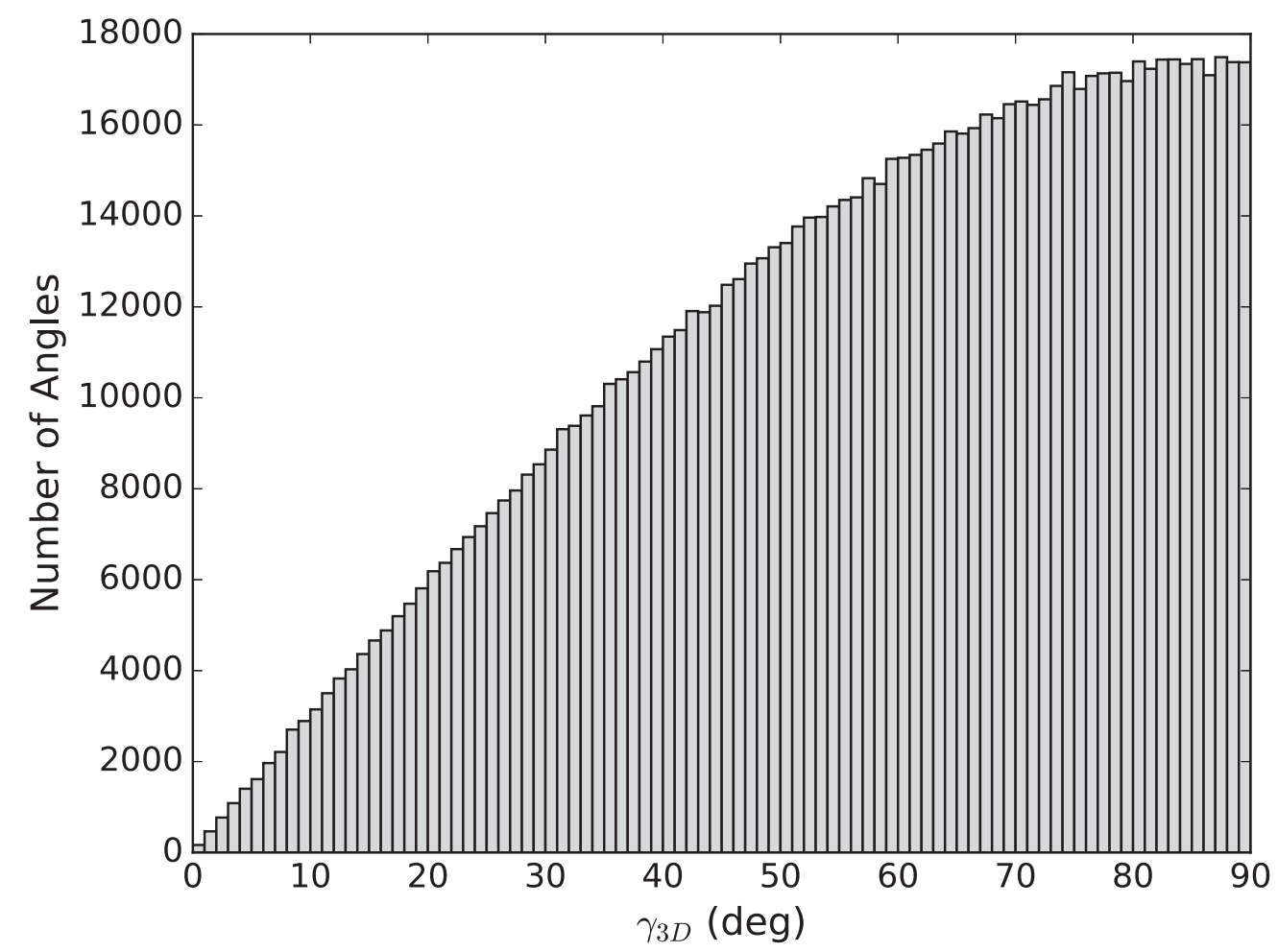

Figure 14. Histogram of $\gamma_{3 \mathrm{D}}$ for a Monte Carlo simulation of $N=10^{6}$ vector pairs. Histogram bin widths are $1^{\circ}$. This histogram shows the approximate shape of the distribution of all possible angles between two vectors in a unit sphere.

distribution of projected outflow-filament angles, $\gamma$, that favors outflows and filaments that are generally perpendicular rather than random. When comparing a random distribution to the AW08 distribution of $\gamma$, the $\mathrm{AD}$ test $p$-value is 0.017 , indicating a significantly non-random distribution. AW08 also found that, if they assumed $\gamma$ follows a tapered Gaussian (i.e., between $0^{\circ}$ and $90^{\circ}$ ) centered at perpendicular, $72 \%$ of the time the outflow is within $45^{\circ}$ of being perpendicular to the filament.

To identify the PA of the outflow, AW08 connected a line between a near-IR identified YSO and the corresponding Herbig Haro Object from Reipurth (1999). The PA of the filaments are determined from flux maps of various submillimeter surveys using SExtractor in STARLINK (with a visual confirmation of the PA). AW08 acknowledged a few selection effects that may bias their results. Specifically, they assumed that all objects have random inclinations, though adjacent sources may have correlated inclinations. Our study also suffers from this bias. AW08 also suggested that they are inherently more likely to find perpendicular outflows since Herbig Haro objects are more likely to be extincted if they are coincident with the filament. For these reasons, they call their conclusion not statistically robust.

AW08 also have some other disadvantages with their data set. Their measured outflow angles rely primarily on published catalogs rather than the physical images. For about half of their sources, they interpreted multiple Herbig Haro objects emitting from a young stellar object as independent outflows. However, upon further analysis, we find that this interpretation is not always accurate. As an example, Figure 15 shows a three-color Spitzer image of the outflow emanating from the SVS 13 protostellar region. The Spitzer image shows only one obvious bipolar outflow from the protostar (greenish $4.5 \mu \mathrm{m}$ color), and the molecular $\mathrm{CO}(1-0)$ line observations confirm that this is a single outflow (contours in Figure 15; Plunkett et al. 2013). However, AW08 declared that the five $\mathrm{HH}$ objects associated with this outflow are five separate outflows, and each of these had a measurement of $\gamma$ above $45^{\circ}$. Therefore, AW08 sometimes have multiple measurements for $\gamma$ for a single outflow, which will significantly bias their results toward a non-random distribution. Moreover, Figure 15 shows that significantly different measurements for $\mathrm{PA}_{\mathrm{Out}}$ can be made for each Herbig Haro object for the same outflow. The dispersion of $\mathrm{HH}$ objects about the outflow lobe may occur due to a precessing outflow coupled with episodic ejections (e.g., Arce \& Goodman 2001; Arce et al. 2010) and/or due to the structure (e.g., clumpiness) of the ambient cloud. Therefore, measuring $\mathrm{PA}_{\text {Out }}$ from Herbig Haro objects alone can result in large $\mathrm{PA}_{\mathrm{Out}}$ measurement errors. AW08 also rely on previous published protostellar positions based on near-IR observations, and these objects sometimes are not the source of the outflows. Figure 15 shows an example of this protostellar misidentification, where AW08 use the location of SVS 13B as the origin of the outflow (marked in red), whereas interferometry data from Plunkett et al. (2013) show that the outflow originates from SVS 13A (marked in blue; coincides with $3 \mathrm{~mm}$ continuum emission). AW08's errant location of the protostar causes the outflow PA to be mis-measured in SVS 13 by up to $\sim 50^{\circ}$.

Given that AW08 have these significant biases and shortcomings in their study, we believe the tentative evidence by AW08 of preferentially perpendicular (and non-random) outflows and filaments is unreliable. 


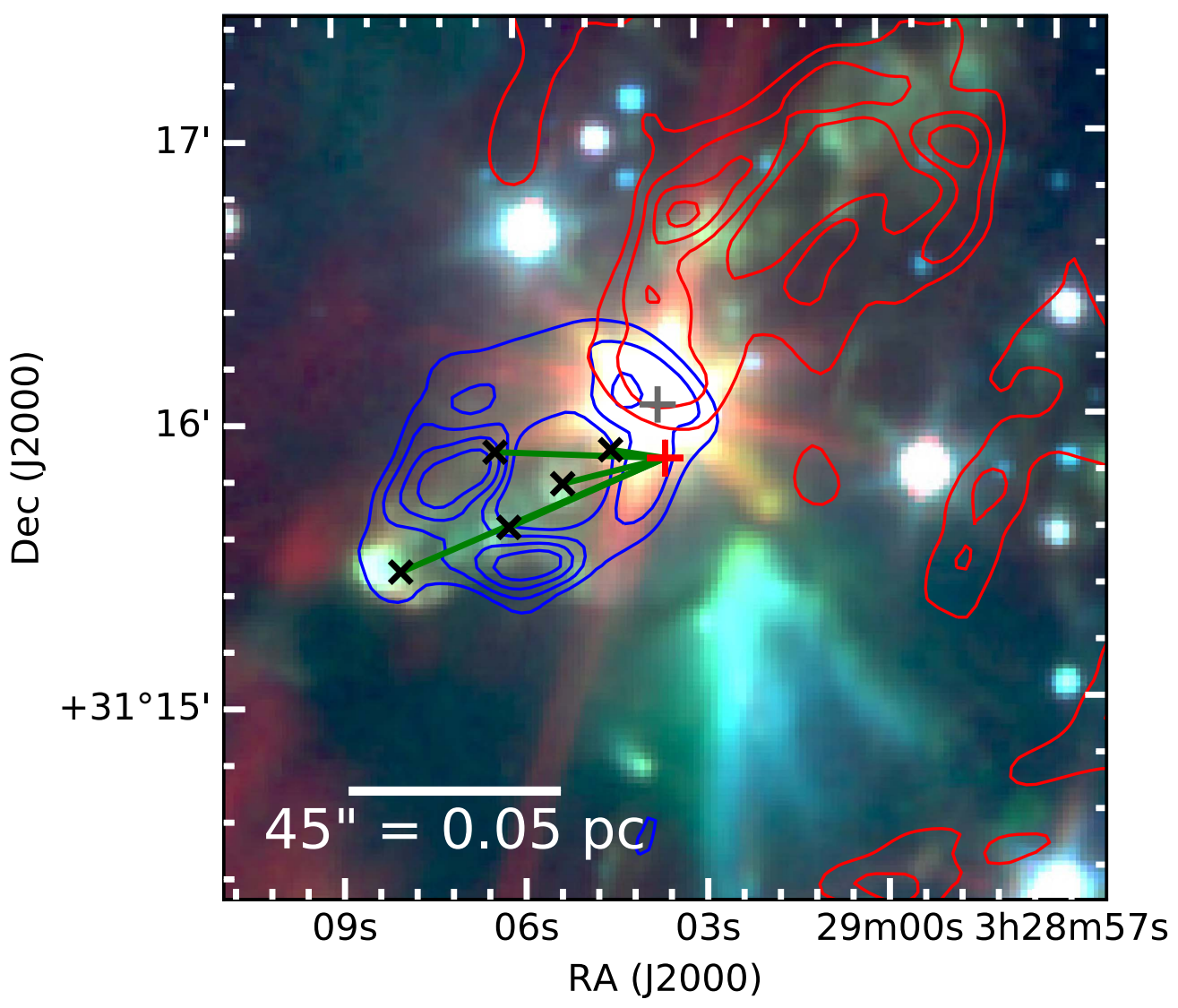

Figure 15. Spitzer three-color image of the SVS 13 star-forming region in NGC1333, with blue, green, and red showing 3.6, 4.5, and $8.0 \mu \mathrm{m}$, respectively. The $\mathrm{CO}(1-0)$ integrated intensity maps from Plunkett et al. (2013) are shown as blue and red contours for the blue and red lobes, respectively. The red plus sign shows the Anathpindika \& Whitworth (2008) position of the protostar based on published near-IR data, while the gray plus sign shows the actual position of the outflow ejection from SVS 13A (based on $3 \mathrm{~mm}$ continuum emission, Plunkett et al. 2013). Black crosses show the locations of Herbig Haro objects identified in Reipurth (1999). The green lines show five outflows identified in Anathpindika \& Whitworth (2008), though it is likely that only one outflow produces all these Herbig Haro objects (Plunkett et al. 2013).

\section{ORCID iDs}

Ian W. Stephens (iD https://orcid.org/0000-0003-3017-4418 Michael M. Dunham (iD https://orcid.org/0000-00030749-9505

Philip C. Myers (1) https://orcid.org/0000-0002-2885-1806

Riwaj Pokhrel (iD https://orcid.org/0000-0002-0557-7349

Sarah I. Sadavoy (iD https://orcid.org/0000-0001-7474-6874

Eduard I. Vorobyov (i) https://orcid.org/0000-0002-6045-0359

John J. Tobin (1D https://orcid.org/0000-0002-6195-0152

Jaime E. Pineda (1) https://orcid.org/0000-0002-3972-1978

Stella S. R. Offner (1D https://orcid.org/0000-0003-1252-9916

Lars E. Kristensen (i) https://orcid.org/0000-0003-1159-3721

Jes K. Jørgensen (i) https://orcid.org/0000-0001-9133-8047

Alyssa A. Goodman (iD https://orcid.org/0000-0003-

1312-0477

Héctor G. Arce (iD https://orcid.org/0000-0001-5653-7817

Adele L. Plunkett (ib https://orcid.org/0000-0002-9912-5705

\section{References}

Abreu-Vicente, J., Stutz, A., Henning, Th., et al. 2017, A\&A, 604, 65 Anathpindika, S., \& Whitworth, A. P. 2008, A\&A, 487, 605

André, P., Men'shchikov, A., Bontemps, S., et al. 2010, A\&A, 518, L102

Arce, H. G., Borkin, M. A., Goodman, A. A., Pineda, J. E., \& Halle, M. W. 2010, ApJ, 715, 1170

Arce, H. G., \& Goodman, A. A. 2001, ApJ, 554, 132

Arzoumanian, D., André, P., Didelon, P., et al. 2011, A\&A, 529, L6
Auddy, S., Basu, S., \& Kudoh, T. 2016, ApJ, 831, 46

Banerjee, R., Pudritz, R. E., \& Anderson, D. W. 2006, MNRAS, 373, 1091 Bertin, E., \& Arnouts, S. 1996, A\&AS, 117, 393

Blum, H. 1967, in Models for the Perception of Speech and Visual Form, ed.

W. Wathen-Dunn (Cambridge, MA: MIT Press), 362

Bodenheimer, P. 1995, ARA\&A, 33, 199

Chen, C.-Y., \& Ostriker, E. C. 2014, ApJ, 785, 69

Chen, C.-Y., \& Ostriker, E. C. 2015, ApJ, 810, 126

Chen, H., Myers, P. C., Ladd, E. F., \& Wood, D. O. S. 1995, ApJ, 445, 377

Chen, X., Arce, H. G., Zhang, Q., et al. 2010, ApJ, 715, 1344

Clark, S. E., Peek, J. E. G., \& Putman, M. E. 2014, ApJ, 789, 82

Clarke, S. D., Whitworth, A. P., Duarte-Cabral, A., \& Hubber, D. A. 2017, MNRAS, 468, 2489

Davis, C. J., Froebrich, D., Stanke, T., et al. 2009, A\&A, 496, 153

Engmann, S., \& Cousineau, D. 2011, J. Applied Quantitative Methods, 6, 1

Enoch, M. L., Evans, N. J., II, Sargent, A. I., \& Glenn, J. 2009, ApJ, 692, 973

Federrath, C. 2016, MNRAS, 457, 375

Feigelson, E. D., \& Babu, G. J. 1992, ApJ, 397, 55

Fogerty, E. L., Carroll-Nellenback, J., Frank, A., Heitsch, F., \& Pon, A. 2017, MNRAS, 470, 2938

Goodman, A. A., Benson, P. J., Fuller, G. A., \& Myers, P. C. 1993, ApJ, 406,528

Hacar, A., Tafalla, M., Kauffmann, J., \& Kovács, A. 2013, A\&A, 554, A55

Heyer, M. H. 1988, ApJ, 324, 311

Hirota, T., Bushimata, T., Choi, Y. K., et al. 2008, PASJ, 60, 37

Ho, P. T. P., Moran, J. M., \& Lo, K. Y. 2004, ApJL, 616, L1

Hou, A., Parker, L. C., Harris, W. E., \& Wilman, D. J. 2009, ApJ, 702, 1199

Hull, C. L. H., Plambeck, R. L., Bolatto, A. D., et al. 2013, ApJ, 768, 159

Hull, C. L. H., Plambeck, R. L., Kwon, W., et al. 2014, ApJS, 213, 13

Ioannidis, G., \& Froebrich, D. 2012, MNRAS, 421, 3257

Isobe, T., Feigelson, E. D., Akritas, M. G., \& Babu, G. J. 1990, ApJ, 364, 104

Koch, E. W., \& Rosolowsky, E. W. 2015, MNRAS, 452, 3435 
Lee, J. W. Y., Hull, C. L. H., \& Offner, S. S. R. 2017, ApJ, 834, 201

Lee, K. I., Dunham, M. M., Myers, P. C., et al. 2015, ApJ, 814, 114

Lee, K. I., Dunham, M. M., Myers, P. C., et al. 2016, ApJL, 820, L2

Looney, L. W., Tobin, J. J., \& Kwon, W. 2007, ApJL, 670, L131

Marsaglia, G. 1972, Ann. Math. Statist., 43, 645

Matthews, B. C., \& Wilson, C. D. 2000, ApJ, 531, 868

McKee, C. F., \& Ostriker, E. C. 2007, ARA\&A, 45, 565

Mouschovias, T. C., \& Morton, S. A. 1985, ApJ, 298, 205

Myers, A. T., Klein, R. I., Krumholz, M. R., \& McKee, C. F. 2014, MNRAS, 439, 3420

Myers, P. C., Fuller, G. A., Goodman, A. A., \& Benson, P. J. 1991, ApJ, 376, 561

Offner, S. S. R., Dunham, M. M., Lee, K. I., Arce, H. G., \& Fielding, D. B. 2016, ApJL, 827, L11

Pereyra, A., \& Magalhães, A. M. 2004, ApJ, 603, 584

Pineda, J. E., Arce, H. G., Schnee, S., et al. 2011a, ApJ, 743, 201

Pineda, J. E., Goodman, A. A., Arce, H. G., et al. 2010, ApJL, 712, L116

Pineda, J. E., Goodman, A. A., Arce, H. G., et al. 2011b, ApJL, 739, L2

Pineda, J. E., Offner, S. S. R., Parker, R. J., et al. 2015, Natur, 518, 213

Plunkett, A. L., Arce, H. G., Corder, S. A., et al. 2013, ApJ, 774, 22

Razali, N. M., \& Wah, Y. B. 2011, J. Statistical Modeling Analytics, 2, 21

Reipurth, B. 1999, A General Catalogue of Herbig-Haro Objects (2nd ed.; Boulder, CO: Univ. Colorado Press), http://casa.colorado.edu/hhcat
Robitaille, T., \& Bressert, E. 2012, APLpy: Astronomical Plotting Library in Python, Astrophysics Source Code Library, ascl:1208.017

Sadavoy, S. I., Di Francesco, J., André, P., et al. 2014, ApJL, 787, L18

Santos, F. P., Busquet, G., Franco, G. A. P., Girart, J. M., \& Zhang, Q. 2016, ApJ, 832, 186

Sault, R. J., Teuben, P. J., \& Wright, M. C. H. 1995, in ASP Conf. Ser. 77 Astronomical Data Analysis Software and Systems IV, ed. R. A. Shaw, H. E. Payne, \& J. J. E. Hayes (San Francisco, CA: ASP), 433

Stephens, M. A. 1974, J. Am. Stat. Assoc., 69, 730

Tatematsu, K., Ohashi, S., Sanhueza, P., et al. 2016, PASJ, 68, 24

Tilley, D. A., \& Pudritz, R. E. 2004, MNRAS, 353, 769

Tobin, J. J., Looney, L. W., Li, Z.-Y., et al. 2016, ApJ, 818, 73

Walch, S., Naab, T., Whitworth, A., Burkert, A., \& Gritschneder, M. 2010, MNRAS, 402, 2253

Weisstein, E. W. 2017, From MathWorld (Oxfordshire: Wolfram Research), http://mathworld.wolfram.com/SpherePointPicking.html

Whitworth, A. P., Chapman, S. J., Bhattal, A. S., et al. 1995, MNRAS, 277,727

Young, K. E., Young, C. H., Lai, S.-P., Dunham, M. M., \& Evans, N. J., II 2015, AJ, 150, 40

Zari, E., Lombardi, M., Alves, J., Lada, C. J., \& Bouy, H. 2016, A\&A, 587, A106 